KA-THEP-4-1995

\title{
Fermionic decays of neutral MSSM Higgs bosons at the one-loop level"
}

\author{
A. Dabelstein' \\ Institut für Theoretische Physik \\ Universität Karlsruhe \\ Kaiserstr. 12 \\ D-76128 Karlsruhe, Germany
}

\begin{abstract}
The results of a complete one-loop calculation for the fermionic decay width $\Gamma\left(h^{0}, H^{0}, A^{0} \rightarrow f \bar{f}\right)$ of the neutral MSSM Higgs bosons are presented and the dominant light Higgs decay channel $h^{0} \rightarrow b \bar{b}$ is discussed in detail. The enhancement of $\Gamma\left(h^{0} \rightarrow b \bar{b}\right)$ compared to the standard Higgs decay is shown for pseudoscalar masses $M_{A} \leq 300 \mathrm{GeV}$, where the one-loop contributions in the MSSM and SM are different. Simpler approximation formulae for the Higgs decays are given and their quality is discussed by introducing an effective neutral scalar mixing angle $\sin ^{2} \alpha_{\text {eff }}$. Finally the Higgs branching ratios in $b \bar{b}, c \bar{c}, \tau^{+} \tau^{-}$are calculated.
\end{abstract}

\footnotetext{
* Supported in part by the European Union under contract CHRX-CT92-0004.

${ }^{1}$ E-Mail: ADD@DMUMPIWH.BITNET
} 


\section{Introduction}

Supersymmetry is at present the most predictive framework for physics beyond the standard model [1]. One theoretical motivation is the cancellation of quadratically divergent contributions to the mass of the scalar Higgs particle. This problem of naturalness is solved in supersymmetric theories. Supersymmetric models allow the unification of gauge couplings at the GUT scale $\mathcal{O}\left(10^{15} \mathrm{GeV}\right)$ [2].

The minimal supersymmetric standard model (MSSM) is considered as the most general supersymmetric extension of the standard model (SM) at low energies [3]. The Higgs sector of the MSSM is that of a 2-Higgs-doublet model, where the coefficients of the Higgs potential are restricted by supersymmetry. As a consequence of the supersymmetric Higgs potential, a light Higgs boson exists with a tree level upper mass bound given by the $Z^{0}$ mass. Radiative corrections to the Higgs mass spectrum, however, predict an upper limit of the light Higgs mass $\mathcal{O}(130 \mathrm{GeV})$ [4, 5]. Calculations were performed at the one-loop level using renormalization group technique [6], effective potential approximation [7] and one-loop calculations with top and stop contributions [8, 9]. Two-loop effects to the upper limit of the lightest Higgs boson mass are discussed in [10.

Production and decay properties of the Higgs boson are charcteristic quantities for the experimental Higgs search at LEP 2, at a $500 \mathrm{GeV} e^{+} e^{-}$collider and LHC. Precise predictions of these quantities require the inclusion of radiative corrections. The one-loop Higgs production cross section and their respective branching ratios (decay width) at $e^{+} e^{-}$ and $p p$ colliders may allow to distinguish between a standard or MSSM Higgs sector. As a first step, complete on-shell renormalization schemes for the MSSM Higgs sector were presented [8, 9, 11, 12].

In this article the complete one-loop partial decay width of the neutral MSSM Higgs bosons $h^{0}\left(H^{0}, A^{0}\right) \rightarrow f \bar{f}$ is calculated within the on-shell scheme 12]. The dominant fermionic decay width of the light MSSM Higgs boson is discussed in detail and the branching ratios of the light scalar Higgs $h^{0}$ in $b \bar{b}, c \bar{c}, \tau^{+} \tau^{-}$are presented. The discussion points out the differences of the MSSM and the standard Higgs decay width and branching ratios.

Section 2 presents an overview of the Higgs production and decay mechanisms, including radiative corrections. The fermionic decay width with full one-loop corrections is 
calculated in section 3. Finally the numerical results for the decay width and branching ratios are discussed in section 4 for QED/QCD, gluino and weak MSSM virtual contributions. Vertex corrections and self energies are given in the appendix.

\section{Decay channels of the neutral Higgs boson}

Once a Higgs boson is found, it is of importance to investigate its characteristic decay properties. The observed cross section is composed by the production cross section for $e^{+} e^{-} \rightarrow Z h(H)$ or $e^{+} e^{-} \rightarrow A h(H)$ and the branching ratios for the subsequent decays of the scalar bosons. The decay width (respectively the branching ratios) as well as the mass-width correlation are the quantities to differentiate between Higgs bosons of various origin. In the following we briefly review the decay modes of the neutral MSSM Higgs particles and discuss the most important fermionic decays in some more detail. Except from a small part of the parameter space, the fermionic decays are the only decay modes of the light Higgs allowed at tree level. The bosonic decays $H^{0} \rightarrow Z Z, W W$ (which are dominant above the threshold in case of the standard Higgs) are kept small also for increasing $M_{H^{0}}$ by the factor $\cos ^{2}(\alpha-\beta)$.

In Tab. 1 we list the various decay channels of the neutral Higgs bosons indicating the level of the theoretical predictions by:

full electroweak: complete 1-loop electroweak calculation performed and available

QCD: QCD corrections performed and available

improved Born: decay width is calculated including the complete 1-loop scalar 2point functions.

The signature $\circ$ denotes the corresponding decay mode as proceeding through 1-loop in lowest order.

\section{$3 \quad$ Fermionic Higgs decays}

\subsection{Tree level structure}

The Higgs sector of the MSSM consists of two scalar doublets $H_{1}, H_{2}$ with opposite hypercharge $Y_{1}=-Y_{2}=-1$ and vacuum expectation values $v_{1}, v_{2}$ [1, 12]. The Higgs potential contains two independent free parameters, which can conveniently be chosen as $\tan \beta=v_{2} / v_{1}$ and $M_{A}$, where $M_{A}$ is the mass of the $A^{0}$ boson.

In lowest order the Yukawa coupling of the standard (MSSM) Higgs bosons to fermions reads:

$$
T_{H f f}=-\frac{i e m_{f}}{2 s_{W} M_{W}} \cdot \kappa_{H}^{f}
$$


Table 1: Decay channels of the neutral Higgs bosons

\begin{tabular}{|c|c|c|c|c|c|c|c|}
\hline & $f \bar{f}$ & $Z Z$ & $Z_{\gamma}$ & $\gamma \gamma$ & $h h$ & $A A$ & $Z h$ \\
\hline$h^{0} \rightarrow$ & $\begin{array}{l}\text { full } \mathrm{EW}^{1)} \\
\mathrm{QCD}^{3)}\end{array}$ & full $\mathrm{EW}^{2)}$ & $\begin{array}{l}\text { o full } \mathrm{EW}^{2)} \\
\mathrm{QCD}^{4)}\end{array}$ & $\begin{array}{l}\text { f full EW } \mathrm{EW}^{2)} \\
\mathrm{QCD}^{5)}\end{array}$ & - & full $\mathrm{EW}^{2)}$ & - \\
\hline$H^{0} \rightarrow$ & $\begin{array}{l}\text { full } \mathrm{EW}^{1)} \\
\mathrm{QCD}^{3)}\end{array}$ & full $\left.\mathrm{EW}^{2}\right)$ & $\begin{array}{l}\text { o full } \mathrm{EW}^{2)} \\
\mathrm{QCD}^{4)}\end{array}$ & $\begin{array}{l}\circ \text { full EW }{ }^{2)} \\
\text { QCD }^{5}\end{array}$ & full $\mathrm{EW}^{2)}$ & full $\mathrm{EW}^{2)}$ & - \\
\hline$A^{0} \rightarrow$ & $\begin{array}{l}\text { full } \mathrm{EW}^{1)} \\
\mathrm{QCD}^{3)}\end{array}$ & $\circ$ full $\mathrm{EW}^{2)}$ & o full $\mathrm{EW}^{2)}$ & $\circ$ full $\mathrm{EW}^{2)}$ & - & - & full $\mathrm{EW}^{2)}$ \\
\hline & $\overline{\bar{\chi} \tilde{\chi} \tilde{\chi}}$ & 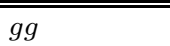 & $\overline{\bar{g} \bar{g} \tilde{g}}$ & & & & \\
\hline$h^{0} \rightarrow$ & $\begin{array}{l}\text { improved } \\
\text { Born }^{6)}\end{array}$ & $\begin{array}{l}\circ \text { full } \mathrm{EW}^{2)} \\
\mathrm{QCD}^{5)}\end{array}$ & $\circ \mathrm{QCD}^{7)}$ & & & & \\
\hline$H^{0} \rightarrow$ & $\begin{array}{l}\text { improved } \\
\text { Born }^{6)}\end{array}$ & $\begin{array}{l}\circ \text { full } \mathrm{EW}^{2)} \\
\mathrm{QCD}^{5)}\end{array}$ & $\left.\circ \mathrm{QCD}^{7}\right)$ & & & & \\
\hline$A^{0} \rightarrow$ & $\begin{array}{l}\text { improved } \\
\text { Born }^{6)}\end{array}$ & $\begin{array}{l}\circ \text { full } \mathrm{EW}^{2)} \\
\mathrm{QCD}^{5)}\end{array}$ & $\circ \mathrm{QCD}^{7)}$ & & & & \\
\hline
\end{tabular}

1) Dabelstein, Hollik

2) Chankowski, Pokorski, Rosiek 11, 13,

3) Braaten, Leveille 14]; Bardin et al. 115]; Drees, Hikasa [16], Chankowski et al. 13]

4) Djouadi, Spira, van der Bij, Zerwas [17]

5) Djouadi, Spira, Zerwas 18]

6) Gunion, Haber [19], improved by the Higgs 2-point functions from Chankowski, Pokorski, Rosiek [13]

7) Ng, Pois, Yuan; Djouadi, Drees 20]

where the weak mixing angle $s_{W}=\sin \theta_{W}$ is introduced in the convention of $s_{W}^{2}=$ $1-M_{W}^{2} / M_{Z}^{2}$ [21]. The coefficients $\kappa_{H}^{f}$ are listed in Tab. 目 for the neutral Higgs particles of the standard model $(H=S M)$ and the MSSM $\left(H=h^{0}, H^{0}, A^{0}\right)$. In lowest order, the

\begin{tabular}{|c|c|c|c|c|}
\hline$\kappa_{H}^{f}$ & $H=$ SM-Higgs & $H=h^{0}$ & $H=H^{0}$ & $H=A^{0}$ \\
\hline$f=u$ & 1 & $\frac{\cos \alpha}{\sin \beta}$ & $\frac{\sin \alpha}{\sin \beta}$ & $-i \cot \beta \gamma_{5}$ \\
\hline$f=d$ & 1 & $-\frac{\sin \alpha}{\cos \beta}$ & $\frac{\cos \alpha}{\cos \beta}$ & $-i \tan \beta \gamma_{5}$ \\
\hline
\end{tabular}

Table 2: Coefficients of the $H \rightarrow f \bar{f}$ vertex

partial decay width $H \rightarrow f \bar{f}$ of a neutral Higgs boson $H$ can be written in the following way:

$$
\Gamma_{0}(H \rightarrow f \bar{f})=\frac{N_{C} G_{F} m_{f}^{2}}{4 \sqrt{2} \pi} \tilde{\beta}^{n} m_{H}\left|\kappa_{H}^{f}\right|^{2},
$$

where $n=3,3,3,1$ for $H=H_{S M}, h^{0}, H^{0}, A^{0}$. In the following $H$ always denotes one of 
the neutral Higgs particles, and

$$
\tilde{\beta}=\sqrt{1-\frac{4 m_{f}^{2}}{m_{H}^{2}}} .
$$

$G_{F}$ is the Fermi constant, related to $M_{W}$ by

$$
G_{F}=\frac{\pi \alpha}{\sqrt{2} s_{W}^{2} M_{W}^{2}} \cdot \frac{1}{1-\Delta r},
$$

where $\Delta r$ is the (SM or MSSM) radiative correction to the $\mu^{-}$decay amplitude [22].

Radiative corrections in the MSSM Higgs sector modify the tree-level decay rate Eq. (3.2) substantially, with the main effect from loops involving the top quark and its scalar partner $\tilde{t}$. The complete decay width comprises the following radiative corrections to

(i) the physical neutral scalar MSSM Higgs

(ii) the full one-loop decay amplitude $H \rightarrow f \bar{f}$

(iii) $\Delta r$ in the MSSM.

\subsection{One-loop structure $H \rightarrow f \bar{f}$}

In this article the one-loop decay amplitudes $H \rightarrow f \bar{f}$ are calculated within the complete one-loop renormalization scheme for the MSSM Higgs sector described in [12]. The one-loop decay widths for $h^{0}, H^{0}, A^{0} \rightarrow f \bar{f}$ with full electroweak MSSM corrections read:

$$
\begin{aligned}
\Gamma_{1}\left(h^{0} \rightarrow f \bar{f}\right)= & \frac{N_{C} G_{F} m_{f}^{2}}{4 \sqrt{2} \pi} M_{h^{0}} \tilde{\beta}^{3}\left|\kappa_{h^{0}}^{f}\right|^{2} . \\
& Z_{h^{0}}\left(\left|1+Z_{h^{0} H^{0}} \frac{\kappa_{H^{0}}^{f}}{\kappa_{h^{0}}^{f}}\right|^{2}+2 \Re e\left(1+Z_{h^{0} H^{0}} \frac{\kappa_{H^{0}}^{f}}{\kappa_{h^{0}}^{f}}\right) \Delta T_{h^{0}}\right)\left(1-\Delta r_{M S S M}\right) \\
\Gamma_{1}\left(H^{0} \rightarrow f \bar{f}\right)= & \frac{N_{C} G_{F} m_{f}^{2}}{4 \sqrt{2} \pi} M_{H^{0}} \tilde{\beta}^{3}\left|\kappa_{H^{0}}^{f}\right|^{2} . \\
& Z_{H^{0}}\left(\left|1+Z_{H^{0} h^{0}} \frac{\kappa_{h^{0}}^{f}}{\kappa_{H^{0}}^{f}}\right|^{2}+2 \Re e\left(1+Z_{H^{0} h^{0}} \frac{\kappa_{h^{0}}^{f}}{\kappa_{H^{0}}^{f}}\right) \Delta T_{H^{0}}\right)\left(1-\Delta r_{M S S M}\right) \\
\Gamma_{1}\left(A^{0} \rightarrow f \bar{f}\right)= & \frac{N_{C} G_{F} m_{f}^{2}}{4 \sqrt{2} \pi} M_{A^{0}} \tilde{\beta}\left|\kappa_{A^{0}}^{f}\right|^{2}\left(1+2 \Re e \Delta T_{A^{0}}\right)\left(1-\Delta r_{M S S M}\right) .
\end{aligned}
$$


$Z_{h^{0}}$ and $Z_{H^{0}}$ are finite wave function renormalizations of the external light and heavy Higgs particles:

$$
\begin{aligned}
Z_{h^{0}} & =\operatorname{Res}_{M_{h^{0}}} \Delta_{h^{0}}=\left.\frac{1}{1+\hat{\Sigma}_{h^{0}}^{\prime}\left(k^{2}\right)-\left(\frac{\hat{\Sigma}_{h^{0} H^{0}}^{2}\left(k^{2}\right)}{k^{2}-m_{H^{0}}^{2}+\hat{\Sigma}_{H^{0}}\left(k^{2}\right)}\right)^{\prime}}\right|_{k^{2}=M_{h^{0}}^{2}} \\
Z_{H^{0}} & =\operatorname{Res}_{M_{H^{0}}} \Delta_{H^{0}}=\left.\frac{1}{1+\hat{\Sigma}_{H^{0}}^{\prime}\left(k^{2}\right)-\left(\frac{\hat{\Sigma}_{h^{0} H^{0}}^{2}\left(k^{2}\right)}{k^{2}-m_{h^{0}}^{2}+\hat{\Sigma}_{h^{0}}\left(k^{2}\right)}\right)^{\prime}}\right|_{k^{2}=M_{H^{0}}^{2}},
\end{aligned}
$$

where $\Delta_{h^{0}}, \Delta_{H^{0}}$ are the diagonal $h^{0}, H^{0}$-propagators and $\hat{\Sigma}_{h^{0}}, \hat{\Sigma}_{H^{0}}, \hat{\Sigma}_{h H}$ are the renormalized $h^{0}, H^{0}$ self energies and mixing [12]. The $h^{0}-H^{0}$ mixing enters in terms of

$$
\begin{aligned}
Z_{h^{0} H^{0}} & =-\frac{\hat{\Sigma}_{h^{0} H^{0}}\left(M_{h^{0}}^{2}\right)}{M_{h^{0}}^{2}-m_{H^{0}}^{2}+\hat{\Sigma}_{H^{0}}\left(M_{h^{0}}^{2}\right)} \\
Z_{H^{0} h^{0}} & =-\frac{\hat{\Sigma}_{h^{0} H^{0}}\left(M_{H^{0}}^{2}\right)}{M_{H^{0}}^{2}-m_{h^{0}}^{2}+\hat{\Sigma}_{h^{0}}\left(M_{H^{0}}^{2}\right)} .
\end{aligned}
$$

The pseudoscalar Higgs self energy and $A^{0} G^{0}$ mixing in the decay width $\Gamma_{1}\left(A^{0} \rightarrow f \bar{f}\right)$, Eq. (3.4), do not contribute, because the renormalization condition sets the residue of the pseudoscalar Higgs propagator equal to one. The Slavnov-Taylor identity yields:

$$
k^{2} \hat{\Sigma}^{A^{0} Z^{0}}\left(k^{2}\right)-M_{Z} \hat{\Sigma}^{A^{0} G^{0}}\left(k^{2}\right)=0,
$$

where $\hat{\Sigma}^{A^{0} Z^{0}}\left(M_{A}^{2}\right)=0$ [12].

The renormalized vertex correction $\Delta T_{H}$ in Eq. (3.4) is the sum of the one-loop vertex diagrams $\Delta T_{i}$ given in appendix $\mathrm{A}$ and a counterterm $\mathrm{CT}$ :

$$
\Delta T_{h^{0}, H^{0}, A^{0}}=\sum_{i=1}^{N}\left(\frac{\alpha}{4 \pi} \Delta T_{i}\right)_{h^{0}, H^{0}, A^{0}}+C T .
$$

The counterterm CT reads:

$$
C T=\frac{\delta m_{f}}{m_{f}}+\frac{\delta Z_{L}^{f}+\delta Z_{R}^{f}}{2}+\frac{\delta v}{v},
$$

with the non-universal contribution from the fermion self energies (App. A):

$$
\frac{\delta m_{f}}{m_{f}}+\frac{\delta Z_{L}^{f}+\delta Z_{R}^{f}}{2}=\Sigma_{S}^{f}\left(m_{f}^{2}\right)-2 m_{f}^{2}\left(\Sigma_{S}^{\prime}\left(m_{f}^{2}\right)+\Sigma_{V}^{\prime}\left(m_{f}^{2}\right)\right),
$$

and the universal part:

$$
\begin{aligned}
2 \frac{\delta v}{v}= & 2 \frac{\delta v_{i}}{v_{i}}=-\Sigma_{A}^{\prime}\left(M_{A}^{2}\right)+\frac{\tan \beta-\cot \beta}{M_{Z}} \Sigma_{A Z}\left(M_{A}^{2}\right)- \\
& \left(-\Sigma_{\gamma}^{\prime}(0)+2 \frac{s_{W}}{c_{W}} \frac{\Sigma_{\gamma Z}(0)}{M_{Z}^{2}}+\frac{c_{W}^{2}}{s_{W}^{2}} \frac{\Sigma_{Z}\left(M_{Z}^{2}\right)}{M_{Z}^{2}}-\frac{c_{W}^{2}-s_{W}^{2}}{s_{W}^{2}} \frac{\Sigma_{W}\left(M_{W}^{2}\right)}{M_{W}^{2}}\right) .
\end{aligned}
$$




\subsection{The one-loop mixing angle $\sin \alpha$}

Introducing universal one-loop coupling coefficients via

$$
\begin{aligned}
\kappa_{h^{0}, 1-\text { loop }}^{f} & =\sqrt{Z_{h^{0}}}\left(\kappa_{h^{0}}^{f}+Z_{h^{0} H^{0}} \kappa_{H^{0}}^{f}\right) \\
\kappa_{H^{0}, 1-\text { loop }}^{f} & =\sqrt{Z_{H^{0}}}\left(\kappa_{H^{0}}^{f}+Z_{H^{0} h^{0}} \kappa_{h^{0}}^{f}\right),
\end{aligned}
$$

an effective universal one-loop mixing angle $\alpha_{1-\text { loop }}$ can be defined through the coefficients in Tab. 2 :

$$
\sin ^{2} \alpha_{1-\text { loop }}=\cos ^{2} \beta \cdot\left(\kappa_{h^{0}, 1-\text { loop }}^{f}\right)^{2}, f=b \text { and }-\pi / 2 \leq \alpha_{1-\text { loop }} \leq 0 .
$$

A good approximation for this mixing angle $\alpha_{1-\text { loop }}$, including only the leading oneloop term

$$
\begin{aligned}
\omega_{t}=\frac{N_{C} G_{F} m_{t}^{4}}{\sqrt{2} \pi^{2} \sin ^{2} \beta} & \left(\log \left(\frac{m_{\tilde{t}_{1}} m_{\tilde{t}_{2}}}{m_{t}^{2}}\right)+\frac{A_{t}\left(A_{t}+\mu \cot \beta\right)}{m_{\tilde{t}_{1}}^{2}-m_{\tilde{t}_{2}}^{2}} \log \frac{m_{\tilde{t}_{1}}^{2}}{m_{\tilde{t}_{2}}^{2}}\right. \\
& \left.+\frac{A_{t}^{2}\left(A_{t}+\mu \cot \beta\right)^{2}}{\left(m_{\tilde{t}_{1}}^{2}-m_{\tilde{t}_{2}}^{2}\right)^{2}}\left(1-\frac{m_{\tilde{t}_{1}}^{2}+m_{\tilde{t}_{2}}^{2}}{m_{\tilde{t}_{1}}^{2}-m_{\tilde{t}_{2}}^{2}} \log \frac{m_{\tilde{t}_{1}}}{m_{\tilde{t}_{2}}}\right)\right) \\
\lambda_{t}=\frac{N_{C} G_{F} m_{t}^{4}}{\sqrt{2} \pi^{2} \sin ^{2} \beta} & \left(\frac{\mu\left(A_{t}+\mu \cot \beta\right)}{m_{\tilde{t}_{1}}^{2}-m_{\tilde{t}_{2}}^{2}} \log \frac{m_{\tilde{t}_{1}}^{2}}{m_{\tilde{t}_{2}}^{2}}\right. \\
& \left.+\frac{2 \mu A_{t}\left(A_{t}+\mu \cot \beta\right)^{2}}{\left(m_{\tilde{t}_{1}}^{2}-m_{\tilde{t}_{2}}^{2}\right)^{2}}\left(1-\frac{m_{\tilde{t}_{1}}^{2}+m_{\tilde{t}_{2}}^{2}}{m_{\tilde{t}_{1}}^{2}-m_{\tilde{t}_{2}}^{2}} \log \frac{m_{\tilde{t}_{1}}}{m_{\tilde{t}_{2}}}\right)\right) \\
\sigma_{t}=\frac{N_{C} G_{F} m_{t}^{4}}{\sqrt{2} \pi^{2} \sin ^{2} \beta} & \frac{\mu^{2}\left(A_{t}+\mu \cot \beta\right)^{2}}{\left(m_{\tilde{t}_{1}}^{2}-m_{\tilde{t}_{2}}^{2}\right)^{2}}\left(1-\frac{m_{\tilde{t}_{1}}^{2}+m_{\tilde{t}_{2}}^{2}}{m_{\tilde{t}_{1}}^{2}-m_{\tilde{t}_{2}}^{2}} \log \frac{m_{\tilde{t}_{1}}}{m_{\tilde{t}_{2}}}\right),
\end{aligned}
$$

follows from the diagonalization of the one-loop Higgs mass matrix [23]

$$
\mathcal{M}_{\text {Higgs }}=\frac{\sin 2 \beta}{2}\left(\begin{array}{ll}
\cot \beta M_{Z}^{2}+\tan \beta M_{A}^{2}+\sigma_{t} & -M_{Z}^{2}-M_{A}^{2}+\lambda_{t} \\
-M_{Z}^{2}-M_{A}^{2}+\lambda_{t} & \tan \beta M_{Z}^{2}+\cot \beta M_{A}^{2}+\omega_{t}
\end{array}\right) .
$$

This approximate effective mixing angle $\alpha_{\text {eff }}$ is determined by

$$
\tan \alpha_{e f f}=\frac{-\left(M_{A}^{2}+M_{Z}^{2}-\lambda_{t}\right) \tan \beta}{M_{Z}^{2}+M_{A}^{2} \tan ^{2} \beta+\sigma_{t} \tan \beta-\left(1+\tan ^{2} \beta\right) M_{h^{0}, \text { eff }}^{2}},
$$

where $M_{h^{0}, \text { eff }}$ is the solution for the light Higgs mass [0] :

$$
\begin{aligned}
M_{H, h, e f f}^{2}= & \frac{M_{A}^{2}+M_{Z}^{2}+\omega_{t}+\sigma_{t}}{2} \pm\left(\frac{\left(M_{A}^{2}+M_{Z}^{2}\right)^{2}+\left(\omega_{t}-\sigma_{t}\right)^{2}}{4}-M_{A}^{2} M_{Z}^{2} \cos ^{2} 2 \beta\right. \\
& \left.+\frac{\left(\omega_{t}-\sigma_{t}\right) \cos 2 \beta}{2}\left(M_{A}^{2}-M_{Z}^{2}\right)-\frac{\lambda_{t} \sin 2 \beta}{2}\left(M_{A}^{2}+M_{Z}^{2}\right)+\frac{\lambda_{t}^{2}}{4}\right)^{1 / 2} .
\end{aligned}
$$


The mixing angle $\alpha_{e f f}$ corresponds to the effective potential approach with top and stop contributions.

Incorporating also the other correction terms of Eq. (3.4), a complete one-loop effective mixing angle $\alpha_{f}$ can be defined

$$
\begin{aligned}
& \frac{\Gamma_{1}\left(h^{0} \rightarrow f \bar{f}\right) \cos ^{2} \beta}{\Gamma_{S M, 0}(h \rightarrow f \bar{f})}=\sin ^{2} \alpha_{1-\text { loop }}+\delta_{V}^{f}-\Delta r_{M S S M} \equiv \sin ^{2} \alpha_{f} \quad, \quad\left(I_{3}^{f}=-1 / 2\right) \\
& \frac{\Gamma_{1}\left(h^{0} \rightarrow f \bar{f}\right) \sin ^{2} \beta}{\Gamma_{S M, 0}(h \rightarrow f \bar{f})}=\cos ^{2} \alpha_{1-\text { loop }}+\delta_{V}^{f}-\Delta r_{M S S M} \equiv \cos ^{2} \alpha_{f} \quad, \quad\left(I_{3}^{f}=+1 / 2\right)
\end{aligned}
$$

with the full one-loop width $\Gamma_{1}$, Eq. (3.4), normalized to the standard tree level width $\Gamma_{S M, 0}$. Besides the universal mixing angle $\alpha_{1}$, defined in Eq. (3.11), $\Delta r_{M S S M}$ is another universal contribution, whereas the residual, mainly vertex correction $\delta_{V}^{f}$ is fermion specific making the complete one-loop effective mixing angle flavour dependent. A momentum dependent mixing angle $\alpha$ has been defined in [24].

For $f=b, \sin ^{2} \alpha_{b}$ is shown in Figs. 1 a,b as a function of the renormalized light Higgs mass $M_{h^{0}}$ and for fixed $\tan \beta$ values. Together with the complete result (dashed line), the approximations in Eqs. (3.11, 3.14) are also shown (dotted, full). In the range $\tan \beta \leq 30$ the full calculation is about $8 \%$ below the approximation (3.14). Figs. 1 c,d plot $\sin ^{2} \alpha$ as a function of the pseudoscalar mass $M_{A}$ and with the same set of parameters as in Figs. 1 a,b. The presentation in Figs. 1 c,d shows the complete result and the approximations $\sin ^{2} \alpha_{e f f}, \sin ^{2} \alpha_{1-\text { loop }}$ for large pseudoscalar mass $M_{A}>150 \mathrm{GeV}$ more precisely than Figs. 1 a,b in the Higgs mass range near the upper limit of the light Higgs mass $M_{h^{0}}$.

\section{Discussion}

Radiative corrections to the partial decay width $H \rightarrow f \bar{f}$ include QED/QCD, weak MSSM and virtual gluino contributions. The following subsections discuss these corrections and present the numerical sizes individually. QED/QCD corrections are identical with the standard Higgs decay $H_{S M} \rightarrow f \bar{f}$ contributions. Weak MSSM contributions give sizeable contributions to the partial decay width of the neutral light and heavy Higgs boson in the intermediate pseudoscalar Higgs mass range $M_{A}$. Vertex corrections with virtual gluino exchange are discussed separatly for the decay channel $h^{0}\left(H^{0}, A^{0}\right) \rightarrow f \bar{f}$. Finally the branching ratios $h^{0} \rightarrow b \bar{b}, c \bar{c}, \tau^{+} \tau^{-}$are presented. 


\subsection{QED and QCD corrections}

QED/QCD corrections to the partial decay width $H \rightarrow f \bar{f}$ appear through vertex diagrams with virtual photons/gluons and final state photon/gluon radiation [14, 15, 16]:

$$
\Delta \Gamma_{Q E D}=\Delta \Gamma_{Q E D}^{V}+\Delta \Gamma_{Q E D}^{B}=\Gamma_{1} \cdot \delta_{Q E D},
$$

where $\delta_{Q E D}$ for the scalar Higgs bosons reads:

$$
\begin{aligned}
\delta_{Q E D}^{S} & =\frac{\alpha}{\pi} Q_{f}^{2} \Delta_{S} \\
\Delta_{S} & =\frac{A(\tilde{\beta})}{\tilde{\beta}}+\frac{3+34 \tilde{\beta}^{2}-13 \tilde{\beta}^{4}}{16 \tilde{\beta}^{3}} \log \frac{1+\tilde{\beta}}{1-\tilde{\beta}}+\frac{3\left(-1+7 \tilde{\beta}^{2}\right)}{8 \tilde{\beta}^{2}} .
\end{aligned}
$$

The pseudoscalar Higgs decay width $A \rightarrow f \bar{f}$ receives a QED correction $\delta_{Q E D}$ different from Eq. (4.2), [16 :

$$
\begin{aligned}
\delta_{Q E D}^{P} & =\frac{\alpha}{\pi} Q_{f}^{2} \Delta_{P} \\
\Delta_{P} & =\frac{A(\tilde{\beta})}{\tilde{\beta}}+\frac{19+2 \tilde{\beta}^{2}+3 \tilde{\beta}^{4}}{16 \tilde{\beta}} \log \frac{1+\tilde{\beta}}{1-\tilde{\beta}}+\frac{3\left(7-\tilde{\beta}^{2}\right)}{8},
\end{aligned}
$$

where

$$
\begin{aligned}
A(\tilde{\beta})= & \left(1+\tilde{\beta}^{2}\right) \cdot \\
& \cdot\left[4 \operatorname{Li}_{2}\left(\frac{1-\tilde{\beta}}{1+\tilde{\beta}}\right)+2 L i_{2}\left(-\frac{1-\tilde{\beta}}{1+\tilde{\beta}}\right)-3 \log \frac{2}{1+\tilde{\beta}} \log \frac{1+\tilde{\beta}}{1-\tilde{\beta}}-2 \log \tilde{\beta} \log \frac{1+\tilde{\beta}}{1-\tilde{\beta}}\right] \\
& -3 \tilde{\beta} \log \frac{4}{1-\tilde{\beta}^{2}}-4 \tilde{\beta} \log \tilde{\beta}
\end{aligned}
$$

In the region $m_{f}^{2} \ll M_{H}^{2}$ the QED correction for both scalar and pseudoscalar Higgs particles, sufficiently accurate, is given by:

$$
\delta_{Q E D} \simeq \frac{\alpha}{\pi} Q_{f}^{2}\left[-3 \log \left(\frac{M_{H}}{m_{f}}\right)+\frac{9}{4}\right] .
$$

The renormalization group improved QCD corrected decay width reads [14]:

$$
\Gamma_{1, Q C D}=\Gamma_{1} \cdot \frac{m_{q}^{2}\left(M_{H}^{2}\right)}{m_{q, 0}^{2}}\left[1+\frac{\alpha_{s}\left(M_{H}^{2}\right)}{\pi} C_{F}\left(\Delta_{S, P}+3 \log \frac{M_{H}}{m_{q, 0}}\right)\right],
$$


where $m_{q}\left(M_{H}^{2}\right)$ is the effective quark mass from the renormalization group equation 25

$$
\begin{aligned}
m_{q}\left(q^{2}\right)= & m_{q, 0}\left(\frac{\beta_{0} \alpha_{s}\left(q^{2}\right)}{2 \pi}\right)^{-\gamma_{0} / 2 \beta_{0}}\left[1+\frac{\beta_{1} \gamma_{0}-\beta_{0} \gamma_{1}}{\beta_{0}^{2}} \frac{\alpha_{s}\left(q^{2}\right)}{8 \pi}\right. \\
& \left.+\left(\frac{\left(\beta_{1} \gamma_{0}-\beta_{0} \gamma_{1}\right)^{2}}{2 \beta_{0}^{4}}+\frac{\gamma_{0}\left(\beta_{2} \beta_{0}-\beta_{1}^{2}\right)}{\beta_{0}^{3}}+\frac{\gamma_{1} \beta_{1}}{\beta_{0}^{2}}-\frac{\gamma_{2}}{\beta_{0}}\right)\left(\frac{\alpha_{s}\left(q^{2}\right)}{8 \pi}\right)^{2}+\ldots\right]
\end{aligned}
$$

and $m_{q}\left(m_{q, 0}^{2}\right)=m_{q, 0}$ is the on-shell mass. The coefficients in Eq. (4.7) are:

$$
\begin{aligned}
& \beta_{0}=\frac{33-2 N_{f}}{3} \\
& \beta_{1}=102-\frac{38}{3} N_{f} \\
& \beta_{2}=\frac{2857}{2}-\frac{5033}{18} N_{f}+\frac{325}{54} N_{f}^{2} \\
& \gamma_{0}=-8 \\
& \gamma_{1}=-\frac{404}{3}+\frac{40}{9} N_{f} \\
& \gamma_{2}=\frac{2}{3}\left[\frac{140}{27} N_{f}^{2}+\left(160 \zeta(3)+\frac{2216}{9}\right) N_{f}-3747\right]
\end{aligned}
$$

where $\zeta(3) \approx 1.2020596 \ldots$ and $N_{f}=6$ for $q^{2}>4 m_{t}^{2}$. The strong coupling constant $\alpha_{s}$ with three-loop contributions is given by:

$$
\alpha_{s}\left(q^{2}\right)=\frac{4 \pi}{\beta_{0} L_{q}}\left[1-\frac{\beta_{1}}{\beta_{0}^{2}} \frac{\log L_{q}}{L_{q}}+\frac{\beta_{1}^{2}}{\beta_{0}^{4}} \frac{\log ^{2} L_{q}}{L_{q}^{2}}-\frac{\beta_{1}^{2}}{\beta_{0}^{4}} \frac{\log L_{q}}{L_{q}^{2}}+\frac{\beta_{2} \beta_{0}-\beta_{1}^{2}}{\beta_{0}^{4}} \frac{1}{L_{q}^{2}}\right]
$$

where $L_{q}=\log \left(q^{2} / \Lambda_{Q C D, N_{f}}^{2}\right)$ and $\Lambda_{Q C D}^{N_{f}=5}=150 \mathrm{MeV} . \Lambda_{Q C D}^{N_{f}=6}$ follows from the condition:

$$
\alpha_{s}^{(5)}\left(m_{t}\right)=\alpha_{s}^{(6)}\left(m_{t}\right)
$$

The QED corrections are small for $b$-quarks $\left(-\delta_{Q E D}<0.4 \%\right.$ for $M_{H}$ up to $\left.1 \mathrm{TeV}\right)$ and somewhat bigger for $c$-quarks $(<1.8 \%)$ and $\tau$-leptons $(<4 \%)$. QED contributions to top quark decays are $\leq 0.4 \%$ for $M_{H}>500 \mathrm{GeV}$. Near the top production threshold, the Coulomb singularity appears and non-perturbative effects have to be taken into account (we do not consider these subtleties here). The fermionic decay width for a scalar Higgs vanishes for $M_{H} \rightarrow 2 m_{t}$ [16], whereas for the pseudoscalar Higgs a finite contribution remains.

The QCD corrections to the hadronic decay width in terms of the on-shell masses are large: in the $b \bar{b}(c \bar{c})$ channel, $\delta_{Q C D}=-39 \%$ for a light Higgs $<2 M_{W}$, increasing up to 
$-60 \%(-75 \%)$ for $1 \mathrm{TeV}$ Higgs boson. The dominant part can be absorbed in the running quark masses. For $M_{H}$ sufficiently above the $t \bar{t}$ threshold, the QCD corrections to the $H \rightarrow t \bar{t}$ width are typically $\delta_{Q C D} \leq 15 \%$.

\subsection{Weak MSSM corrections}

The partial decay width $h^{0} \rightarrow b \bar{b}$ is the dominant decay channel for a light scalar MSSM Higgs and a standard Higgs with a mass below $M_{H}<140 \mathrm{GeV}$. A precise prediction of the $H \rightarrow b \bar{b}$ decay width and the fermionic $b \bar{b}, c \bar{c}, \tau^{+} \tau^{-}$branching ratios requires the inclusion of radiative corrections at the one-loop level. One-loop contributions to the $b \bar{b}$ decay channel are discussed in detail for the light (heavy) scalar and pseudoscalar Higgs decay width $h^{0}\left(H^{0}, A^{0}\right) \rightarrow b \bar{b}$. The $c \bar{c}, \tau^{+} \tau^{-}$decay channels are presented within the context of the Higgs branching ratios in the following subsection.

The one-loop contributions from the residual MSSM particles are contained in the electroweak decay width $\Gamma_{1}$, Eq. (3.4). Figs. 2 a,b show the one-loop decay width $\Gamma_{1}$ for the light Higgs decay channel $h^{0} \rightarrow b \bar{b}$ as a function of the light Higgs mass $M_{h^{0}}$. In Fig. 2 a, $\tan \beta=2,30$ while $\tan \beta=0.5,8$ values are presented in Fig. 2 b. Soft breaking parameters are $m_{s f}=700 \mathrm{GeV}, \mu=100 \mathrm{GeV}, M=550 \mathrm{GeV}$ and no mixing of left-right sfermion states is assumed. The sfermion mass matrix is given in Eq. (4.13). Appendix B contains the chargino and neutralino mass matrix. The $h^{0} \rightarrow b \bar{b}$ decay width dependence on the top-quark mass $m_{t}$ is shown for $m_{t}=160 \mathrm{GeV}$ (dotted line), $m_{t}=175$ $\mathrm{GeV}$ (solid line) and $m_{t}=190 \mathrm{GeV}$ (dashed line), as favoured by the CDF data for the top-quark mass [26]. In Figs. 2 a,b the upper limit of the light Higgs mass $M_{h^{0}}$ increases $\sim m_{t}^{4}$, as discussed in Eq. (3.15). For a light Higgs mass $M_{h^{0}}<80 \mathrm{GeV}$ and $\tan \beta \geq 5$ the partial decay width $h^{0} \rightarrow b \bar{b}$ is almost insensitive on $m_{t} . \Gamma_{1}\left(h^{0} \rightarrow b \bar{b}\right)$ increases with $\sim m_{t}^{4}$ for a light Higgs mass $M_{h^{0}}>80 \mathrm{GeV}$. This $m_{t}^{4}$ dependence of the partial decay width is a universal contribution of the external Higgs two-point functions, described by the one-loop mixing angle $\sin ^{2} \alpha_{e f f}$, in Eq. (3.14). Figs. $2 \mathrm{c}, \mathrm{d}$ show the partial decay width as a function of the pseudoscalar Higgs mass $M_{A}$. The decay width $\Gamma_{1}\left(h^{0} \rightarrow b \bar{b}\right)$ reaches a maximum near $90 \mathrm{GeV}<M_{A}<110 \mathrm{GeV}$ and decreases for $M_{A}>110 \mathrm{GeV}$. Figs. 2 a-d also show the standard Higgs partial decay width $H_{S M} \rightarrow b \bar{b}$ with one-loop weak corrections [15, 27]. The mass $M_{H_{S M}}$ is chosen to be equal the light MSSM Higgs mass $M_{h^{0}}$. In Figs. $2 \mathrm{c}$,d the solid (dashed) standard Higgs decay width corresponds to the MSSM $\tan \beta$ values 30 (2) in Fig. $2 \mathrm{c}$ and 8 (0.5) in Fig. 2 d. As a result, $\Gamma_{1}\left(h^{0} \rightarrow b \bar{b}\right)$ in the MSSM is enhanced for all $\tan \beta$ values compared to the standard decay width. For large pseudoscalar masses $M_{A} \rightarrow \infty$, however, the MSSM decay width approaches 
the standard model result closely. This behaviour is discussed in terms of Eq. (3.11), where $\sin ^{2} \alpha \rightarrow \cos ^{2} \beta$ in the limit $M_{A} \rightarrow \infty$. In Fig. 2 the pseudoscalar Higgs mass range is chosen up to $300 \mathrm{GeV}$. The gap between the MSSM and the standard decay width is sizeable for $M_{A}=300 \mathrm{GeV}$. Larger pseudoscalar masses tend to approach the standard model decay width as can be seen in Figs. 2 c,d. Even in the limit of a large pseudoscalar mass $M_{A}$, the genuine vertex corrections to the MSSM (SM) Higgs decay width are different, due to the presence of virtual supersymmetric particles in Eq. (3.4).

The sfermion mass dependence on $\Gamma_{1}\left(h^{0} \rightarrow b \bar{b}\right)$ is shown in Figs 3 a,b as a function of the light Higgs mass $M_{h^{0}}$ and for $\tan \beta$ values $\tan \beta=2,30$ (Fig. 3 a), $\tan \beta=0.5,8$ (Fig. $3 \mathrm{~b}$ ). No mixing of left-right sfermion states is assumed. In Figs. 3 a,b the sfermion soft breaking parameters are $m_{s f}=1 \mathrm{TeV}$ (solid line), $m_{s f}=500 \mathrm{GeV}$ (dotted), $m_{s f}=300 \mathrm{GeV}$ (short dashed) and $m_{s f}=200 \mathrm{GeV}$ (long dashed). The upper limit of the light Higgs mass and the partial decay width $\Gamma_{1}\left(h^{0} \rightarrow b \bar{b}\right)$ increases $\sim \log \left(\frac{m_{\tilde{t}_{L}} m_{\tilde{t}_{R}}}{m_{t}^{2}}\right)$, Eq. (3.15). Mixing effects from the left and right sfermions states are shown in Fig. 3 c for $\tan \beta=2,30$. The off-diagonal mixing parameter $A_{t}^{\prime}$ in Eq. (4.14) is $A_{t}^{\prime}=0,100$ $\mathrm{GeV}, 200 \mathrm{GeV}, 300 \mathrm{GeV}, 400 \mathrm{GeV}$. These mixings increase the light Higgs $M_{h^{0}}$ and the partial decay width $\Gamma_{1}\left(h^{0} \rightarrow b \bar{b}\right)$ simultaneously.

Effects from gaugino soft breaking parameters $M, \mu$ are displayed in Fig. $3 \mathrm{~d}$. The dependence of the partial decay width $\Gamma_{1}\left(h^{0} \rightarrow b \bar{b}\right)$ on $M$ is shown for $M=100,200$ $\mathrm{GeV}$ to be very small. $\mu$ enters the sfermion mass matrix, Eq. (4.13), in the off-diagonal entries and in the Higgs-sfermion couplings. In Fig. $3 \mathrm{~d}$ no left-right mixing is present by fine-tuning the $A$ parameter. Large $\tan \beta$ values $(\tan \beta \geq 30)$, however, show sizeable effects for the partial decay width, since the parameter $A$ increases with $\tan \beta$, $\mu$ and contributes to the $H-\tilde{f}_{L^{-}}-\tilde{f}_{R}$ couplings. For lower $\tan \beta$ values the partial decay width is almost insensitive on $\mu$.

Figs. 4 a,b show the one-loop partial decay width $\Gamma_{1}$ of the heavy Higgs boson $H^{0} \rightarrow b \bar{b}$ as a function of the heavy Higgs mass $M_{H^{0}}$ for values $\tan \beta=0.5,2,8,30$. The top quark mass dependence of the decay width $\Gamma_{1}(H \rightarrow b \bar{b})$ is presented in Fig. 4 a for $m_{t}=160$ $\mathrm{GeV}$ (dotted line), $175 \mathrm{GeV}$ (solid) and $190 \mathrm{GeV}$ (dashed), while Figs. 4 b shows the decay width for several sfermion soft breaking parameters $m_{s f}=1 \mathrm{TeV}$ (solid line), 500 GeV (dotted), $300 \mathrm{GeV}$ (short dashed) and $200 \mathrm{GeV}$ (long dashed). The soft breaking parameters are described in the figure caption. For a heavy scalar Higgs mass $M_{H^{0}}>180$ $\mathrm{GeV}$ and $\tan \beta>2$ the decay width $\Gamma_{1}$ is almost insensitive on $m_{t} . \Gamma_{1}\left(H^{0} \rightarrow b \bar{b}\right)$ increases for large $\tan \beta$ values. 
The pseudoscalar one-loop decay width $\Gamma_{1}\left(A^{0} \rightarrow b \bar{b}\right)$ is shown in Fig. $4 \mathrm{c}$ for fixed soft breaking parameters and $\tan \beta$ values. The partial decay width $\Gamma_{1}\left(A^{0} \rightarrow f \bar{f}\right)$ for down(up) type fermions increases (decreases) with $\sim \tan ^{2} \beta$ ( $\cot ^{2} \beta$ ). Vertex corrections $\delta \Gamma_{b}=2 \Re e \Delta T_{A^{0}}$ in Eq. (3.4) are shown in Fig. $4 \mathrm{~d}$ as a function of the pseudoscalar mass $M_{A}$. The top quark mass dependence of the vertex corrections is presented for $m_{t}=160,175,190 \mathrm{GeV}$ (dotted, solid, dashed line). The one-loop contributions $\delta \Gamma_{b}$ are large $\approx 15 \%$ for $\tan \beta=0.5$. For $\tan \beta \geq 2, \delta \Gamma_{b} \approx 3-8 \%$ and decreases with larger pseudoscalar masses $M_{A}$.

\subsection{Vertex corrections of virtual gluinos}

The supersymmetric partners of the $S U(3)$ gluons, the gluinos $\tilde{g}_{a}$, appear as virtual states in the $H \rightarrow q \bar{q}$ vertex corrections (together with squarks) with the strong coupling constant $\alpha_{s}$. They contribute a shift $\delta \Gamma_{G l}$ in the decay width for $h^{0}, H^{0}, A^{0} \rightarrow q \bar{q}$.

$$
\Gamma_{1, G l}(H \rightarrow q \bar{q})=\Gamma_{1}(H \rightarrow q \bar{q})\left(1+\delta \Gamma_{G l}\right),
$$

where

$$
\delta \Gamma_{G l}=2 \Re e\left(\Delta T_{G l}^{H}+\Sigma_{S, G l}^{f}\left(m_{q}^{2}\right)-2 m_{q}^{2}\left(\Sigma_{S, G l}^{f^{\prime}}\left(m_{q}^{2}\right)+\Sigma_{V, G l}^{f^{\prime}}\left(m_{q}^{2}\right)\right)\right),
$$

is the one-loop gluino contribution to the decay width $H \rightarrow q \bar{q}$. The vertex correction $\Delta T_{G l}^{H}$ and self energies $\Sigma_{S, G l}^{f}, \Sigma_{V, G l}^{f}$ are given in Eq. (4.18, 4.22).

Mixing effects from virtual squarks in the vertex corrections $\Delta T_{G l}^{H}$ and quarks self energies $\Sigma_{G l}^{f}$ are described by a $2 \times 2$ squark mass matrix:

$$
\mathcal{M}_{\tilde{\mathrm{q}}}^{2}=\left(\begin{array}{ll}
M_{\tilde{\tilde{Q}}}^{2}+m_{q}^{2}+M_{Z}^{2}\left(I_{3}-Q_{q} s_{W}^{2}\right) \cos 2 \beta & m_{q}\left(A_{q}+\mu\{\cot \beta, \tan \beta\}\right) \\
m_{q}\left(A_{q}+\mu\{\cot \beta, \tan \beta\}\right) & M_{\{\tilde{U}, \tilde{D}\}}^{2}+m_{q}^{2}+M_{Z}^{2} Q_{q} s_{W}^{2} \cos 2 \beta
\end{array}\right)
$$

with SUSY soft breaking parameters $M_{\tilde{Q}}, M_{\tilde{U}, \tilde{D}}, A_{q}$, and $\mu$. The notation in the offdiagonal entries in Eq. (4.13):

$$
A_{q}^{\prime}=A_{q}+\mu\{\cot \beta, \tan \beta\}
$$

will be used. In the following discussion the soft breaking parameters are taken to be equal $m_{s f}=M_{\tilde{Q}}=M_{\tilde{U}, \tilde{D}}$. Up and down type squarks in (1.13) are distinguished by setting $\mathrm{f}=\mathrm{u}, \mathrm{d}$ and the $\{u, d\}$ entries in the parenthesis. The parameter $\mu$ in the off-diagonal matrix elements in (4.13) is also present in the gaugino sector. The sfermion masses, obtained from diagonalizing (4.13) are:

$$
m_{\tilde{q}_{i}}^{2}=\frac{1}{2}\left(\operatorname{Tr} \mathcal{M}_{\tilde{\mathrm{q}}}^{2} \pm \sqrt{\left(\operatorname{Tr} \mathcal{M}_{\tilde{\mathrm{q}}}^{2}\right)^{2}-4 \operatorname{Det} \mathcal{M}_{\tilde{\mathrm{q}}}^{2}}\right), \mathrm{i}=1,2,
$$


where the corresponding rotation matrices

$$
U\left(\theta_{\tilde{q}}\right)=\left(\begin{array}{rr}
\cos \theta_{\tilde{q}} & \sin \theta_{\tilde{q}} \\
-\sin \theta_{\tilde{q}} & \cos \theta_{\tilde{q}}
\end{array}\right)
$$

are described by the sfermion mixing angle $\theta_{\tilde{q}}$ :

$$
\tan 2 \theta_{\tilde{q}}=\frac{2 m_{q}\left(A_{q}+\mu\{\cot \beta, \tan \beta\}\right)}{M_{\tilde{q}}^{2}-M_{\{\tilde{U}, \tilde{D}\}}^{2}+M_{Z}^{2}\left(I_{3}-2 Q_{q} s_{W}^{2}\right) \cos 2 \beta} .
$$

The one-loop vertex correction for the scalar neutral Higgs decay $h^{0}\left(H^{0}\right) \rightarrow q \bar{q}$ is given by:

$$
\Delta T_{G l}^{h^{0}\left(H^{0}\right)}=-\frac{\alpha_{s}}{3 \pi} \sum_{i, j=1}^{2} \frac{T_{i, j}^{h^{0}\left(H^{0}\right)}}{T_{h^{0}\left(H^{0}\right) f f}}\left[2 m_{q} \delta_{i j} C_{1}^{+}-m_{\tilde{g l}} \Delta_{i j} C_{0}\right]\left(M_{h^{0}\left(H^{0}\right)}^{2}, m_{\tilde{q}_{i}}, m_{\tilde{q}_{j}}, m_{\tilde{g l}}\right)
$$

where $T_{h^{0}\left(H^{0}\right) f f}$ in Eq. (4.18) are the tree-level couplings, $\delta_{i j}$ is the unit matrix and

$$
\Delta_{i j}=\left(\begin{array}{cc}
\sin 2 \theta_{\tilde{q}} & \cos 2 \theta_{\tilde{q}} \\
\cos 2 \theta_{\tilde{q}} & -\sin 2 \theta_{\tilde{q}}
\end{array}\right)
$$

The pseudoscalar Higgs decay $A^{0} \rightarrow q \bar{q}$ yields the vertex correction:

$$
\Delta T^{A^{0}}=-\frac{\alpha_{s}}{3 \pi} \sum_{i, j=1}^{2} \frac{T_{i, j}^{A^{0}}}{T_{A^{0} f f}} \epsilon_{i j} m_{\tilde{g l}} C_{0}\left(M_{A^{0}}^{2}, m_{\tilde{q}_{i}}, m_{\tilde{q}_{j}}, m_{\tilde{g} l}\right) \gamma_{5}
$$

with $T_{A^{0} f f}$ in Eq. (4.19) from Tab. 2 and $\epsilon_{12}=-\epsilon_{21}=1 . T_{i, j}^{H}$ are the $H-\tilde{q}_{i^{-}} \tilde{q}_{j}$ couplings in the squark mass eigenstate fields, obtained by the transformation:

$$
T_{i, j}^{H}=U\left(\theta_{\tilde{q}}\right)_{i, a} T_{a, b}^{H} U^{\dagger}\left(\theta_{\tilde{q}}\right)_{b, j}
$$

where

$$
\begin{aligned}
T_{a, b}^{h^{0}} & =i g\left(\begin{array}{ll}
\frac{M_{Z}}{c_{W}}\left(I_{3}-Q_{q} s_{W}^{2}\right) \sin (\alpha+\beta)-\frac{m_{q}^{2}\{\cos \alpha,-\sin \alpha\}}{M_{W}\{\sin \beta, \cos \beta\}} & \frac{m_{q}\left(\mu\{\sin \alpha,-\cos \alpha)-A_{q}\{\cos \alpha,-\sin \alpha\}\right)}{2 M_{W}\{\sin \beta, \cos \beta\}} \\
\frac{m_{q}\left(\mu\{\sin \alpha,-\cos \alpha)-A_{q}\{\cos \alpha,-\sin \alpha\}\right)}{2 M_{W}\{\sin \beta, \cos \beta\}} & \frac{M_{Z}}{c_{W}} Q_{q} s_{W}^{2} \sin (\alpha+\beta)-\frac{m_{q}^{2}\{\cos \alpha,-\sin \alpha\}}{M_{W}\{\sin \beta, \cos \beta\}}
\end{array}\right) \\
T_{a, b}^{H^{0}} & =-i g\left(\begin{array}{ll}
\frac{M_{Z}}{c_{W}}\left(I_{3}-Q_{q} s_{W}^{2}\right) \cos (\alpha+\beta)+\frac{m_{q}^{2}\{\sin \alpha, \cos \alpha\}}{M_{W}\{\sin \beta, \cos \beta\}} & \frac{m_{q}\left(\mu\{\cos \alpha, \sin \alpha)+A_{q}\{\sin \alpha, \cos \alpha\}\right)}{2 M_{W}\{\sin \beta, \cos \beta\}} \\
\frac{m_{q}\left(\mu\{\cos \alpha, \sin \alpha)+A_{q}\{\sin \alpha, \cos \alpha\}\right)}{2 M_{W}\{\sin \beta, \cos \beta\}} & \frac{M_{Z}}{c_{W}} Q_{q} s_{W}^{2} \cos (\alpha+\beta)+\frac{m_{q}^{2}\{\sin \alpha, \cos \alpha\}}{M_{W}\{\sin \beta, \cos \beta\}}
\end{array}\right) \\
T_{a, b}^{A^{0}} & =g\left(\begin{array}{ll}
0 & -\frac{m_{q}}{2 M_{W}}\left(\mu-A_{q}\{\cot \beta, \tan \beta\}\right) \\
\frac{m_{q}}{2 M_{W}}\left(\mu-A_{q}\{\cot \beta, \tan \beta\}\right) & 0
\end{array}\right) .
\end{aligned}
$$


The first (second) column in the parentheses belongs to the up (down) squark coupling. In Eq. (4.12) the fermion self energy with virtual gluinos and squarks for the scalar (vector) components reads:

$$
\begin{aligned}
\Sigma_{S}^{f}\left(m_{q}^{2}\right) & =\frac{-\alpha_{s}}{3 \pi} \frac{m_{\tilde{g} l}}{m_{q}} \sin 2 \theta_{\tilde{q}}\left(B_{0}\left(m_{q}^{2}, m_{\tilde{g l}}, m_{\tilde{q}_{1}}\right)-B_{0}\left(m_{q}^{2}, m_{\tilde{g} l}, m_{\tilde{q}_{2}}\right)\right) \\
\Sigma_{V, i}^{f}\left(m_{q}^{2}\right) & =\frac{-\alpha_{s}}{3 \pi} \sum_{i=1}^{2} B_{1}\left(m_{q}^{2}, m_{\tilde{g l}}, m_{\tilde{q}_{i}}\right) .
\end{aligned}
$$

The integrals $B_{0}, B_{1}, C_{0}, C_{1}$ are defined in appendix A.

The numerical analysis of the gluino contributions $\delta \Gamma_{G l}$ is shown in Fig. 5. Light (heavy) scalar and pseudoscalar Higgs boson decays $H \rightarrow b \bar{b}$ are presented in Figs. 5 $(\mathrm{a}, \mathrm{b}),(\mathrm{c}, \mathrm{d}),(\mathrm{e}, \mathrm{f})$. In Figs. 5 a,c,e the one-loop corrections are shown as a function of the Higgs mass and for two fixed gluino mass parameters $m_{\tilde{g l}}=500 \mathrm{GeV}$ (solid line), $200 \mathrm{GeV}$ (dotted line). The sfermion mass is $m_{s f}=700 \mathrm{GeV}$ (solid line), $m_{s f}=500 \mathrm{GeV}$ (dotted line) and no left-right mixing is present. The contribution $\delta \Gamma_{G l}$ decreases for larger gluino masses $m_{\tilde{g} l}$ and larger Higgs masses $M_{H}$. The corrections are sizeable $\simeq 30 \%$ for large $\tan \beta=30$ values and a light mass $M_{h^{0}}$ below the upper mass limit. Mixing effects from left-right sfermion states are shown in Fig. $5 \mathrm{~b}, \mathrm{~d}, \mathrm{f}$ as a function of the $\mu$ parameter and all other parameters fixed. The corrections $\delta \Gamma_{G l}$ are $\sim \mu$ and become large (20\%) for lighter Higgs masses and $|\mu| \geq 250 \mathrm{GeV}$.

\subsection{Fermionic branching ratios $H \rightarrow f \bar{f}$}

Branching ratios of the fermionic Higgs decay channels are experimentally measurable, even if the partial decay width $\Gamma(H \rightarrow f \bar{f})$ can not be measured directly. In the following the branching ratios of the light neutral scalar MSSM Higgs $h^{0}$ and the standard Higgs $H_{S M}$ in $b \bar{b}, c \bar{c}, \tau^{+} \tau^{-}$are presented. Here we restrict the discussion to the fermionic branching ratio $R_{f}$, given by:

$$
R_{f}=\frac{\Gamma_{1}(H \rightarrow f \bar{f})}{\sum_{f=\tau, c, b, t} \Gamma_{1}(H \rightarrow f \bar{f})},
$$

where the light fermion contributions are negligible. Figs. 6 a,b show the light neutral MSSM branching ratios $R_{f}$ for $b \bar{b}$ (Fig. 6 a) and the $c \bar{c}, \tau^{+} \tau^{-}$decay channels (Fig. 6 b), where the full one-loop contributions from section 4.2 are included. No QED/QCD and gluino contributions are included in the figure. In Fig. 6 the branching ratio $R_{f}$ is a function of the light Higgs mass $M_{h^{0}}$ and values $\tan \beta=0.5,2,8,30$ are shown for a top-quark mass $m_{t}=175 \mathrm{GeV}$ and soft breaking parameters $m_{s f}=700 \mathrm{GeV}, \mu=100$ 
$\mathrm{GeV}, M=550 \mathrm{GeV}$. The $h^{0} \rightarrow b \bar{b}$ decay rate is $87-95 \%$ for $0.5 \leq \tan \beta \leq 30 . R_{f}$ decreases for the $b$ and $\tau$ decay channels (increases for $c$ ) near the upper limit of the light MSSM Higgs mass. In the limit $M_{A} \rightarrow \infty$ the branching ratio $R_{f}$ reaches the standard model result closely, as shown by the dotted lines in Figs. 6 a,b. Deviations from the standard model result are model dependent supersymmetric vertex contributions. $b$ and $\tau$ decay ratios $R_{f}$ are between $0-9 \%$ and $4-6 \%$ in the range $0.5 \leq \tan \beta \leq 30$.

The branching ratio $R_{f}$ in Eq. (4.23), where the approximation formulae Eq. (3.14) for the partial decay width is used instead:

$$
\Gamma_{1}^{\prime}(H \rightarrow f \bar{f})=\frac{N_{C} G_{F} m_{f}^{2}}{4 \sqrt{2} \pi} M_{H} \tilde{\beta}^{n}\left|\kappa_{H, e f f}^{f}\right|^{2},
$$

with $\kappa_{h^{0}, \text { eff }}^{d}=-\frac{\sin \alpha_{e f f}}{\cos \beta}, \ldots$ yields a qualitative good prediction within $0.1 \%$ for $\tan \beta \geq 2$ and $0.6 \%$ for $\tan \beta=0.5$ compared to the complete result. The approximate result Eq. (4.24) is plotted by the dashed line. In the ratio $R_{f}$, Eq. (4.23), the universal contributions $\Delta r$ and the vertex correction part $\delta v / v$ from the one-loop decay width Eq. (3.4) cancel. Therefore the complete result for the branching ratio $R_{f}$ and the approximation formulae, Eq. (4.24) are in good agreement.

\section{Conclusions}

The fermionic partial decay width $\Gamma_{1}(H \rightarrow f \bar{f})$ for the neutral MSSM Higgs bosons $h^{0}, H^{0}, A^{0}$ is calculated with full one-loop MSSM contributions for the decay channels $b \bar{b}$, $c \bar{c}, \tau^{+} \tau^{-}$. In the calculation, the renormalization scheme for the supersymmetric Higgs sector 12 was used. The tree-level decay width for down (up) type fermions is enhanced (suppressed) compared to the standard model Higgs decay width. One-loop corrections in the pseudoscalar Higgs mass range $80 \mathrm{GeV} \leq M_{A} \leq 110 \mathrm{GeV}$ give large corrections $\sim m_{t}^{4}$ to the decay width $\Gamma_{1}\left(h^{0}\left(H^{0}\right) \rightarrow f \bar{f}\right)$. The diagonalization of the neutral scalar Higgs mass matrix, described by the mixing angle $\alpha_{1-l o o p}$, receives the dominant contributions from top and stop loops $\mathcal{O}\left(\mathrm{m}_{\mathrm{t}}^{4}\right)$. The mixing angle $\sin ^{2} \alpha$ is calculated with full oneloop contributions $\left(\sin ^{2} \alpha_{1-\text { loop }}\right)$, in the effective potential approximation $\left(\sin ^{2} \alpha_{\text {eff }}\right)$ and as a flavour dependent effective mixing angle $\left(\sin ^{2} \alpha_{f}\right)$. The mixing angles $\sin ^{2} \alpha_{1-\text { loop }}$, $\sin ^{2} \alpha_{\text {eff }}$ and $\sin ^{2} \alpha_{f}$ are in agreement within $8 \%$. For large pseudoscalar Higgs masses $M_{A} \rightarrow \infty$ the decay width $\Gamma_{1}\left(h^{0} \rightarrow f \bar{f}\right)$ approaches the standard model result. In this limit, non-universal model dependent one-loop contributions to the decay width $\Gamma_{1}$ can distinguish between a standard and MSSM Higgs boson and depend in detail on the chosen parameters. Virtual gluino vertex corrections give sizeable contributions to the 
$H \rightarrow b \bar{b}$ decay width (branching ratios). The branching ratios for the light neutral scalar Higgs decay $h^{0} \rightarrow b \bar{b}, c \bar{c}, \tau^{+} \tau^{-}$are presented. The full calculation and the approximation formulae Eq. (4.24) are in agreement within $0.2-0.6 \%$.

\section{Acknowledgements.}

I am grateful to W. Hollik for suggesting this topic and reading the manuscript and to S. Pokorski for various discussions. 


\section{A Vertex corrections and self energies}

The Feynman rules of the minimal supersymmetric standard model are given in [1]. All analytical formulae are calculated in the 't Hooft-Feynman gauge. The two- and three-point functions $B_{0}, B_{1}, B_{22}, C_{0}, C_{1}$ and $C_{2}$ are defined at the end of appendix A. $f^{\prime}$ denotes the isospin partner for the external fermion $f$ in the same isodoublet.

- Scalar MSSM $h^{0}\left(H^{0}\right) \rightarrow f \bar{f}$ vertex corrections*:

$$
\begin{aligned}
& \Delta T_{1}=V_{1}^{s}\left(k^{2}, m_{f}, m_{f}, m_{f}, M_{Z}, v_{f}, a_{f}\right) \\
& \text { with } \quad v_{f}=\frac{I_{3}^{f}-2 s_{W}^{2} Q_{f}}{2 s_{W} c_{W}}, a_{f}=\frac{I_{3}^{f}}{2 s_{W} c_{W}} \\
& \Delta T_{2}=\frac{m_{f^{\prime}}}{8 m_{f} s_{W}^{2}} \frac{\kappa_{H}^{f^{\prime}}}{\kappa_{H}^{f}} V_{1}^{s}\left(k^{2}, m_{f}, m_{f^{\prime}}, m_{f^{\prime}}, M_{W}, 1,-1\right) \\
& \Delta T_{3}=-\frac{m_{f^{\prime}}}{8 M_{W}^{2} m_{f} s_{W}^{2}} \frac{\kappa_{H}^{f^{\prime}}}{\kappa_{H}^{f}} V_{2}^{s}\left(k^{2}, m_{f}, m_{f^{\prime}}, m_{f^{\prime}}, m_{H^{+}}, \lambda_{f}, \mu_{f}\right) \\
& \text { with } \quad \lambda_{f}= \begin{cases}m_{f} \tan \beta+m_{f^{\prime}} \cot \beta & , f=d \\
m_{f^{\prime}} \tan \beta+m_{f} \cot \beta & , f=u\end{cases} \\
& \mu_{f}= \begin{cases}-m_{f} \tan \beta+m_{f^{\prime}} \cot \beta & , f=d \\
+m_{f^{\prime}} \tan \beta-m_{f} \cot \beta & , f=u\end{cases} \\
& \Delta T_{4}=-\frac{m_{f^{\prime}}}{8 M_{W}^{2} m_{f} s_{W}^{2}} \frac{\kappa_{H}^{f^{\prime}}}{\kappa_{H}^{f}} V_{2}^{s}\left(k^{2}, m_{f}, m_{f^{\prime}}, m_{f^{\prime}}, M_{W}, \nu_{f}, \pi_{f}\right) \\
& \text { with } \nu_{f}= \begin{cases}m_{f^{\prime}}-m_{f} & , f=d \\
m_{f}-m_{f^{\prime}} & , f=u\end{cases} \\
& \pi_{f}=\left\{\begin{aligned}
m_{f^{\prime}}+m_{f} & , f=d \\
-m_{f}-m_{f^{\prime}} & , f=u
\end{aligned}\right. \\
& \Delta T_{5}=\frac{m_{f}^{2}\left(\kappa_{H^{0}}^{f}\right)^{2}}{4 M_{W}^{2} s_{W}^{2}} V_{2}^{s}\left(k^{2}, m_{f}, m_{f}, m_{f}, m_{H^{0}}, 1,0\right) \\
& \Delta T_{6}=\frac{m_{f}^{2}\left(\kappa_{h^{0}}^{f}\right)^{2}}{4 M_{W}^{2} s_{W}^{2}} V_{2}^{s}\left(k^{2}, m_{f}, m_{f}, m_{f}, m_{h^{0}}, 1,0\right) \\
& \Delta T_{7}=-\frac{m_{f}^{2}\left|\kappa_{A^{0}}^{f}\right|^{2}}{4 M_{W}^{2} s_{W}^{2}} V_{2}^{s}\left(k^{2}, m_{f}, m_{f}, m_{f}, M_{A^{0}}, 0,1\right)
\end{aligned}
$$

*The upper(lower) line in the parentheses is the $h^{0}\left(H^{0}\right) \rightarrow f \bar{f}$ vertex correction. 


$$
\begin{aligned}
& \Delta T_{8}=-\frac{m_{f}^{2}}{4 M_{W}^{2} s_{W}^{2}} V_{2}^{s}\left(k^{2}, m_{f}, m_{f}, m_{f}, M_{Z}, 0,1\right) \\
& \Delta T_{9}=\frac{M_{W}}{m_{f} s_{W}^{2} \kappa_{H}^{f}} \sum_{i, j, k=1}^{2,2,2} V_{3}^{s}\left(k^{2}, m_{f}, m_{\tilde{\chi}_{j}^{+}}, m_{\tilde{\chi}_{i}^{+}}, m_{\tilde{f}_{k}^{\prime}}, v_{j k}^{f}, a_{j k}^{f}, a_{i k}^{f}, v_{i k}^{f}, O_{i j}^{H}, O_{j i}^{H}\right) \\
& \text { with } \quad v_{i k}^{d, u}= \begin{cases}\left(V_{i 1}, U_{i 1}\right) \cos \tilde{\theta}_{f^{\prime}}-\frac{m_{f^{\prime}}}{\sqrt{2} M_{W}} \frac{V_{i 2}, U_{i 2}}{\sin \beta, \cos \beta} \sin \tilde{\theta}_{f^{\prime}}, k=1 \\
\left(V_{i 1}, U_{i 1}\right) \sin \tilde{\theta}_{f^{\prime}}+\frac{m_{f^{\prime}}}{\sqrt{2} M_{W}} \frac{V_{i 2}, U_{i 2}}{\sin \beta, \cos \beta} \cos \tilde{\theta}_{f^{\prime}}, k=2\end{cases} \\
& a_{i k}^{d, u}= \begin{cases}-\frac{m_{f}}{\sqrt{2} M_{W}} \frac{U_{i 2}, V_{i 2}}{\cos \beta, \sin \beta} \cos \tilde{\theta}_{f^{\prime}} & , k=1 \\
-\frac{m_{f}}{\sqrt{2} M_{W}} \frac{U_{i 2}, V_{i 2}}{\cos \beta, \sin \beta} \sin \tilde{\theta}_{f^{\prime}} & , k=2\end{cases} \\
& O_{i j}^{H}=\frac{1}{\sqrt{2}} \begin{cases}V_{i 1} U_{j 2} \sin \alpha-V_{i 2} U_{j 1} \cos \alpha & , H=h^{0} \\
V_{i 1} U_{j 2} \cos \alpha+V_{i 2} U_{j 1} \sin \alpha & , H=H^{0}\end{cases} \\
& \Delta T_{10}=\frac{2 M_{W}}{m_{f} \kappa_{H}^{f}} \sum_{i, j, k=1}^{4,4,2} V_{3}^{s}\left(k^{2}, m_{f}, m_{\tilde{\chi}_{j}^{0}}, m_{\tilde{\chi}_{i}^{0}}, m_{\tilde{f}_{k}}, v_{j k}^{\prime f}, a_{j k}^{\prime f}, a_{i k}^{\prime f}, v_{i k}^{\prime f}, O_{i j}^{\prime H}, O_{j i}^{\prime H}\right) \\
& \text { with } \quad v_{i k}^{\prime d, u}=\left\{\begin{array}{l}
\left(Q_{f} N_{i 1}^{\prime} \mp \frac{1 / 2+Q_{f} s_{W}^{2}}{s_{W} C_{W}} N_{i 2}^{\prime}\right) \cos \tilde{\theta}_{f}+\frac{m_{f}\left(N_{i 3}, N_{i 4}\right)}{2 M_{W} s_{W}(\cos \beta, \sin \beta)} \sin \tilde{\theta}_{f}, k=1 \\
\left(Q_{f} N_{i 1}^{\prime} \mp \frac{1 / 2 Q_{f} s_{W}^{2}}{s_{W} c_{W}} N_{i 2}^{\prime}\right) \sin \tilde{\theta}_{f}-\frac{m_{f}\left(N_{i 3}, N_{i 4}\right)}{2 M_{W} s_{W}(\cos \beta \sin \beta)} \cos \tilde{\theta}_{f}, k=1
\end{array}\right. \\
& a_{i k}^{\prime d, u}= \begin{cases}\frac{m_{f} N_{i 3}}{2 M_{W} s_{W}(\cos \beta, \sin \beta)} \cos \tilde{\theta}_{f}-\left(Q_{f} N_{i 1}^{\prime}-\frac{Q_{f} s_{W}}{c_{W}} N_{i 2}^{\prime}\right) \sin \tilde{\theta}_{f} \quad, k=1 \\
\frac{m_{f} N_{i 3}}{2 M_{W} s_{W}(\cos \beta, \sin \beta)} \sin \tilde{\theta}_{f}+\left(Q_{f} N_{i 1}^{\prime}-\frac{Q_{f} s_{W}}{c_{W}} N_{i 2}^{\prime}\right) \cos \tilde{\theta}_{f} & , k=2\end{cases} \\
& O_{i j}^{\prime H}=\frac{1}{2} \begin{cases}Q_{i j} \sin \alpha+S_{i j} \cos \alpha & , H=h^{0} \\
Q_{i j} \cos \alpha-S_{i j} \sin \alpha & , H=H^{0}\end{cases} \\
& Q_{i j}=\left(N_{i 3}\left(N_{j 2}-N_{j 1} \tan \theta_{W}\right)+N_{j 3}\left(N_{i 2}-N_{i 1} \tan \theta_{W}\right)\right) / 2 \\
& S_{i j}=\left(N_{i 4}\left(N_{j 2}-N_{j 1} \tan \theta_{W}\right)+N_{j 4}\left(N_{i 2}-N_{i 1} \tan \theta_{W}\right)\right) / 2 \\
& \Delta T_{11}=\frac{1}{4 m_{f} s_{W}^{2} \kappa_{H}^{f}}\left\{\begin{array}{r}
-\cos (\beta-\alpha) \\
\sin (\beta-\alpha)
\end{array}\right\} V_{4}^{s}\left(k^{2}, m_{f}, M_{W}, m_{H^{+}}, m_{f^{\prime}}, 1 / 2,-1 / 2, \lambda_{f}, \mu_{f}\right) \\
& \Delta T_{12}=\frac{1}{2 s_{W} c_{W} \kappa_{H}^{f}}\left\{\begin{array}{r}
\cos (\beta-\alpha) \\
-\sin (\beta-\alpha)
\end{array}\right\} V_{4}^{s}\left(k^{2}, m_{f}, M_{Z}, M_{A}, m_{f}, v_{f}, a_{f}, 0,-1\right) \cdot \\
& \begin{cases}\tan \beta, & f=d \\
\cot \beta, & f=u\end{cases} \\
& \Delta T_{13}=\frac{1}{4 m_{f} s_{W}^{2} \kappa_{H}^{f}}\left\{\begin{array}{c}
\sin (\beta-\alpha) \\
\cos (\beta-\alpha)
\end{array}\right\} V_{4}^{s}\left(k^{2}, m_{f}, M_{W}, M_{W}, m_{f^{\prime}}, 1 / 2,-1 / 2, \nu_{f}, \pi_{f}\right)
\end{aligned}
$$




$$
\begin{aligned}
& \Delta T_{14}=\frac{1}{2 s_{W} c_{W} \kappa_{H}^{f}}\left\{\begin{array}{c}
\sin (\beta-\alpha) \\
\cos (\beta-\alpha)
\end{array}\right\} V_{4}^{s}\left(k^{2}, m_{f}, M_{Z}, M_{Z}, m_{f}, v_{f}, a_{f}, 0,-1\right) . \\
& \left\{\begin{aligned}
1, & f=d \\
-1, & f=u
\end{aligned}\right. \\
& \Delta T_{15}=-\frac{1}{4 m_{f} s_{W}^{2} \kappa_{H}^{f}}\left\{\begin{array}{c}
\sin (\beta-\alpha)+\cos 2 \beta \sin (\beta-\alpha) /\left(2 c_{W}^{2}\right) \\
\cos (\beta-\alpha)-\cos 2 \beta \cos (\beta-\alpha) /\left(2 c_{W}^{2}\right)
\end{array}\right\} . \\
& \text { - } V_{5}^{s}\left(k^{2}, m_{f}, m_{H^{+}}, m_{H^{+}}, m_{f^{\prime}}, \lambda_{f}, \mu_{f}, \lambda_{f},-\mu_{f}\right) \\
& \Delta T_{16}=\frac{m_{f}\left(\kappa_{h^{0}}^{f}\right)^{2}}{4 s_{W}^{2} c_{W}^{2} \kappa_{H}^{f}}\left\{\begin{array}{r}
3 \cos 2 \alpha \sin (\alpha+\beta) \\
2 \sin 2 \alpha \sin (\beta+\alpha)-\cos (\beta+\alpha) \cos 2 \alpha
\end{array}\right\} . \\
& \text { - } V_{5}^{s}\left(k^{2}, m_{f}, m_{h^{0}}, m_{h^{0}}, m_{f}, 1,0,1,0\right) \\
& \Delta T_{17}=\frac{m_{f}\left(\kappa_{H^{0}}^{f}\right)^{2}}{4 s_{W}^{2} c_{W}^{2} \kappa_{H}^{f}}\left\{\begin{array}{r}
-2 \sin 2 \alpha \cos (\beta+\alpha)-\sin (\beta+\alpha) \cos 2 \alpha) \\
3 \cos 2 \alpha \cos (\beta+\alpha)
\end{array}\right\} . \\
& \cdot V_{5}^{s}\left(k^{2}, m_{f}, m_{H^{0}}, m_{H^{0}}, m_{f}, 1,0,1,0\right) \\
& \Delta T_{18}=\frac{m_{f} \sin \alpha \cos \alpha \kappa_{h^{0}}^{f} \kappa_{H^{0}}^{f}}{2 s_{W}^{2} c_{W}^{2} \kappa_{H}^{f}}\left\{\begin{array}{r}
(2 \sin 2 \alpha \sin (\alpha+\beta)-\cos (\alpha+\beta) \cos 2 \alpha) \\
-(2 \sin 2 \alpha \cos (\alpha+\beta)+\sin (\alpha+\beta) \cos 2 \alpha)
\end{array}\right\} . \\
& \cdot V_{5}^{s}\left(k^{2}, m_{f}, m_{h^{0}}, m_{H^{0}}, m_{f}, 1,0,1,0\right) \\
& \Delta T_{19}=-\frac{m_{f} \cos 2 \beta\left(\kappa_{A^{0}}^{f}\right)^{2}}{4 s_{W}^{2} c_{W}^{2} \kappa_{H}^{f}}\left\{\begin{array}{r}
\sin (\beta+\alpha) \\
-\cos (\beta+\alpha)
\end{array}\right\} V_{5}^{s}\left(k^{2}, m_{f}, M_{A}, M_{A}, m_{f}, 0,1,0,1\right) \\
& \Delta T_{20}=\frac{m_{f} \cos 2 \beta}{4 s_{W}^{2} c_{W}^{2} \kappa_{H}^{f}}\left\{\begin{array}{r}
\sin (\beta+\alpha) \\
-\cos (\beta+\alpha)
\end{array}\right\} V_{5}^{s}\left(k^{2}, m_{f}, M_{Z}, M_{Z}, m_{f}, 0,1,0,1\right) \\
& \Delta T_{21}=\frac{-\cos 2 \beta}{8 m_{f} s_{W}^{2} c_{W}^{2} \kappa_{H}^{f}}\left\{\begin{array}{r}
-\sin (\beta+\alpha) \\
\cos (\beta+\alpha)
\end{array}\right\} V_{5}^{s}\left(k^{2}, m_{f}, M_{W}, M_{W}, m_{f^{\prime}}, \nu_{f}, \pi_{f}, \nu_{f},-\pi_{f}\right) \\
& \Delta T_{22}=-\frac{m_{f} \sin 2 \beta}{2 s_{W}^{2} c_{W}^{2} \kappa_{H}^{f}}\left\{\begin{array}{r}
-\sin (\beta+\alpha) \\
\cos (\beta+\alpha)
\end{array}\right\} . \\
& \cdot V_{5}\left(k^{2}, m_{f}, M_{Z}, M_{A}, m_{f}, 0,1,0,1\right) \cdot\left\{\begin{aligned}
\tan \beta & , f=d \\
-\cot \beta & , f=u
\end{aligned}\right. \\
& \Delta T_{23}=\frac{1}{4 M_{W}^{2} m_{f} s_{W}^{2} \kappa_{H}^{f}}\left\{\begin{array}{r}
-\cos (\beta-\alpha)\left(m_{H^{+}}^{2}-m_{h^{0}}^{2}\right) \\
\sin (\beta-\alpha)\left(m_{H^{+}}^{2}-m_{H^{0}}^{2}\right)
\end{array}\right\}
\end{aligned}
$$




$$
\begin{aligned}
& \cdot V_{5}^{s}\left(k^{2}, m_{f}, M_{W}, m_{H^{+}}, m_{f^{\prime}}, \nu_{f}, \pi_{f}, \lambda_{f}, \mu_{f}\right) \\
& \Delta T_{24}=\frac{M_{W}^{2}}{4 m_{f} s_{W}^{2} \kappa_{H}^{f}}\left\{\begin{array}{c}
\sin (\beta-\alpha) \\
\cos (\beta-\alpha)
\end{array}\right\} V_{6}^{s}\left(k^{2}, m_{f}, M_{W}, M_{W}, m_{f^{\prime}}, 1,-1,1,-1\right) \\
& \Delta T_{25}=\frac{2 M_{W}^{2}}{m_{f} c_{W}^{2} \kappa_{H}^{f}}\left\{\begin{array}{c}
\sin (\beta-\alpha) \\
\cos (\beta-\alpha)
\end{array}\right\} V_{6}\left(k^{2}, m_{f}, M_{Z}, M_{Z}, m_{f}, v_{f}, a_{f}, v_{f}, a_{f}\right) \\
& \Delta T_{26}=\frac{M_{W}}{2 s_{W}^{2} m_{f} \kappa_{H}^{f}} \sum_{i=1}^{2}\left[\left(\cos ^{2} \tilde{\theta}_{f^{\prime}} u_{H, 1}+\sin ^{2} \tilde{\theta}_{f^{\prime}} u_{H, 2}+\sin 2 \tilde{\theta}_{f^{\prime}} u_{H, 3}\right)\right. \\
& \cdot V_{5}^{s}\left(k^{2}, m_{f}, m_{\tilde{f}^{\prime}{ }_{1}}, m_{\tilde{f}^{\prime}{ }_{1}}, m_{\tilde{\chi}_{i}^{+}}, v_{i 1}^{f}+a_{i 1}^{f}, v_{i 1}^{f}-a_{i 1}^{f}, v_{i 1}^{f}-a_{i 1}^{f}, v_{i 1}^{f}+a_{i 1}^{f}\right) \\
& +\left(\sin ^{2} \tilde{\theta}_{f^{\prime}} u_{H, 1}+\cos ^{2} \tilde{\theta}_{f^{\prime}} u_{H, 2}-\sin 2 \tilde{\theta}_{f^{\prime}} u_{H, 3}\right) \\
& \cdot V_{5}^{s}\left(k^{2}, m_{f}, m_{\tilde{f}^{\prime}{ }_{2}}, m_{\tilde{f}^{\prime}{ }_{2}}, m_{\tilde{\chi}_{i}^{+}}, v_{i 2}^{f}+a_{i 2}^{f}, v_{i 2}^{f}-a_{i 2}^{f}, v_{i 2}^{f}-a_{i 2}^{f}, v_{i 2}^{f}+a_{i 2}^{f}\right) \\
& +\left(\sin 2 \tilde{\theta}_{f^{\prime}}\left(u_{H, 2}-u_{H, 1}\right)+2 \cos 2 \tilde{\theta}_{f^{\prime}} u_{H, 3}\right) \\
& \left.\cdot V_{5}^{s}\left(k^{2}, m_{f}, m_{\tilde{f}^{\prime}{ }_{1}}, m_{\tilde{f}^{\prime}}, m_{\tilde{\chi}_{i}^{+}}, v_{i 1}^{f}+a_{i 1}^{f}, v_{i 1}^{f}-a_{i 1}^{f}, v_{i 2}^{f}-a_{i 2}^{f}, v_{i 2}^{f}+a_{i 2}^{f}\right)\right] \\
& \Delta T_{27}=\frac{-M_{W}}{m_{f} \kappa_{H}^{f}} \sum_{i=1}^{4}\left[\left(\cos ^{2} \tilde{\theta}_{f} u_{H, 1}+\sin ^{2} \tilde{\theta}_{f} u_{H, 2}+\sin 2 \tilde{\theta}_{f} u_{H, 3}\right)\right. \\
& \cdot V_{5}^{s}\left(k^{2}, m_{f}, m_{\tilde{f}_{1}}, m_{\tilde{f}_{1}}, m_{\tilde{\chi}_{i}^{0}}, v_{i 1}^{\prime f}+a_{i 1}^{\prime f}, v_{i 1}^{\prime f}-a_{i 1}^{\prime f}, v_{i 1}^{\prime f}-a_{i 1}^{\prime f}, v_{i 1}^{\prime f}+a_{i 1}^{\prime f}\right) \\
& +\left(\sin ^{2} \tilde{\theta}_{f} u_{H, 1}+\cos ^{2} \tilde{\theta}_{f} u_{H, 2}-\sin 2 \tilde{\theta}_{f} u_{H, 3}\right) \\
& \cdot V_{5}^{s}\left(k^{2}, m_{f}, m_{\tilde{f}_{2}}, m_{\tilde{f}_{2}}, m_{\tilde{\chi}_{i}^{0}}, v_{i 2}^{\prime f}+a_{i 2}^{\prime f}, v_{i 2}^{\prime f}-a_{i 2}^{\prime f}, v_{i 2}^{\prime f}-a_{i 2}^{\prime f}, v_{i 2}^{\prime f}+a_{i 2}^{\prime f}\right) \\
& +\left(\sin 2 \tilde{\theta}_{f}\left(u_{H, 2}-u_{H, 1}\right)+2 \cos 2 \tilde{\theta}_{f} u_{H, 3}\right) \\
& \left.\cdot V_{5}^{s}\left(k^{2}, m_{f}, m_{\tilde{f}_{1}}, m_{\tilde{f}_{2}}, m_{\tilde{\chi}_{i}^{0}}, v_{i 1}^{\prime f}+a_{i 1}^{\prime f}, v_{i 1}^{\prime f}-a_{i 1}^{\prime f}, v_{i 2}^{\prime f}-a_{i 2}^{\prime f}, v_{i 2}^{\prime f}+a_{i 2}^{\prime f}\right)\right]
\end{aligned}
$$

where

$$
\begin{aligned}
& \left(u_{h^{0}, j}\right)=\left(\begin{array}{l}
\frac{M_{Z}}{c_{W}}\left( \pm \frac{1}{2}-Q_{ \pm} s_{W}^{2}\right) \sin (\alpha+\beta)-\frac{m_{ \pm}^{2}\{\cos \alpha,-\sin \alpha\}}{M_{W}\{\sin \beta, \cos \beta\}} \\
\frac{M_{Z}}{c_{W}} Q_{ \pm} s_{W}^{2} \sin (\alpha+\beta)-\frac{m_{ \pm}^{2}\{\cos \alpha,-\sin \alpha\}}{M_{W}\{\sin \beta, \cos \beta\}} \\
\frac{-m_{f}}{2 M_{W}\{\sin \beta, \cos \beta\}}\left\{\mu \sin \alpha-A_{u} \cos \alpha, \mu \cos \alpha-A_{d} \sin \alpha\right\}
\end{array}\right) \\
& \left(u_{H^{0}, j}\right)=\left(\begin{array}{l}
-\frac{M_{Z}}{c_{W}}\left( \pm \frac{1}{2}-Q_{ \pm} s_{W}^{2}\right) \cos (\alpha+\beta)-\frac{m_{ \pm}^{2}\{\sin \alpha, \cos \alpha\}}{M_{W}\{\sin \beta, \cos \beta\}} \\
-\frac{M_{Z}}{c_{W}} Q_{ \pm} s_{W}^{2} \cos (\alpha+\beta)-\frac{m_{ \pm}^{2}\{\sin \alpha, \cos \alpha\}}{M_{W}\{\sin \beta, \cos \beta\}} \\
\frac{-m_{f}}{2 M_{W}\{\sin \beta, \cos \beta\}}\left\{\mu \cos \alpha+A_{u} \sin \alpha, \mu \sin \alpha+A_{d} \cos \alpha\right\}
\end{array}\right)
\end{aligned}
$$


- Pseudoscalar MSSM $A^{0} \rightarrow f \bar{f}$ vertex correction:

$$
\begin{aligned}
& \Delta T_{1}=V_{1}^{p}\left(k^{2}, m_{f}, m_{f}, m_{f}, M_{Z}, v_{f}, a_{f}\right) \\
& \Delta T_{2}=\frac{m_{f^{\prime}}}{8 m_{f} s_{W}^{2}} \frac{\left|\kappa_{A^{0}}^{f^{\prime}}\right|}{\left|\kappa_{A^{0}}^{f}\right|} V_{1}^{p}\left(k^{2}, m_{f}, m_{f^{\prime}}, m_{f^{\prime}}, M_{W}, 1,-1\right) \\
& \Delta T_{3}=-\frac{m_{f^{\prime}}}{8 M_{W}^{2} m_{f} s_{W}^{2}} \frac{\left|\kappa_{A^{0}}^{f^{\prime}}\right|}{\left|\kappa_{A^{0}}^{f}\right|} V_{2}^{p}\left(k^{2}, m_{f}, m_{f^{\prime}}, m_{f^{\prime}}, m_{H^{+}}, \lambda_{f}, \kappa_{f}\right) \\
& \Delta T_{4}=-\frac{m_{f^{\prime}}}{8 M_{W}^{2} m_{f} s_{W}^{2}} \frac{\left|\kappa_{A^{0}}^{f^{\prime}}\right|}{\left|\kappa_{A^{0}}^{f}\right|} V_{2}^{p}\left(k^{2}, m_{f}, m_{f^{\prime}}, m_{f^{\prime}}, M_{W}, \nu_{f}, \pi_{f}\right) \\
& \Delta T_{5}=\frac{m_{f}^{2}\left(\kappa_{H^{0}}^{f}\right)^{2}}{4 M_{W}^{2} s_{W}^{2}} V_{2}^{p}\left(k^{2}, m_{f}, m_{f}, m_{f}, m_{H^{0}}, 1,0\right) \\
& \Delta T_{6}=\frac{m_{f}^{2}\left(\kappa_{h^{0}}^{f}\right)^{2}}{4 M_{W}^{2} s_{W}^{2}} V_{2}^{p}\left(k^{2}, m_{f}, m_{f}, m_{f}, m_{h^{0}}, 1,0\right) \\
& \Delta T_{7}=-\frac{m_{f}^{2}\left|\kappa_{A^{0}}^{f}\right|^{2}}{4 M_{W}^{2} s_{W}^{2}} V_{2}^{p}\left(k^{2}, m_{f}, m_{f}, m_{f}, M_{A}, 0,1\right) \\
& \Delta T_{8}=-\frac{m_{f}^{2}}{4 M_{W}^{2} s_{W}^{2}} V_{2}^{p}\left(k^{2}, m_{f}, m_{f}, m_{f}, M_{Z}, 0,1\right) \\
& \Delta T_{9}=\frac{M_{W}}{m_{f} s_{W}^{2}\left|\kappa_{A^{0}}^{f}\right|} \sum_{i, j, k=1}^{2,2,2} V_{3}^{p}\left(k^{2}, m_{f}, m_{\tilde{\chi}_{j}^{+}}, m_{\tilde{\chi}_{i}^{+}}, m_{\tilde{f}_{k}^{\prime}}, v_{j k}^{f}, a_{j k}^{f}, a_{i k}^{f}, v_{i k}^{f}, O_{i j}^{A}, Q_{j i}^{A}\right) \\
& \text { with } O_{i j}^{A}=\frac{1}{\sqrt{2}}\left(V_{i 1} U_{j 2} \sin \beta+V_{i 2} U_{j 1} \cos \beta\right)
\end{aligned}
$$$$
\Delta T_{10}=\frac{2 M_{W}}{m_{f}\left|\kappa_{A^{0}}^{f}\right|} \sum_{i, j, j=1}^{4,4,2} V_{3}^{p}\left(k^{2}, m_{f}, m_{\tilde{\chi}_{j}^{0}}, m_{\tilde{\chi}_{i}^{0}}, m_{\tilde{f}_{k}}, v_{j k}^{\prime f}, a_{j k}^{\prime f}, a_{i k}^{\prime f}, v_{i k}^{\prime f}, P_{i j}^{A}, P_{j i}^{A}\right)
$$$$
\text { with } P_{i j}^{A}=\frac{1}{2}\left(Q_{i j} \sin \beta-S_{i j} \cos \beta\right)
$$

$$
\begin{aligned}
\Delta T_{11} & =\frac{1}{4 s_{W}^{2} m_{f}\left|\kappa_{A^{0}}^{f}\right|} V_{4}^{p}\left(k^{2}, m_{f}, M_{W}, m_{H^{+}}, m_{f^{\prime}}, 1 / 2,-1 / 2, \lambda_{f}, \mu_{f}\right) \\
\Delta T_{12} & =\frac{\cos (\beta-\alpha) \kappa_{h^{0}}^{f}}{2 s_{W} c_{W}\left|\kappa_{A^{0}}^{f}\right|} V_{4}^{p}\left(k^{2}, m_{f}, M_{Z}, m_{h^{0}}, m_{f}, v_{f},-a_{f}, 1,0\right) \\
\Delta T_{13} & =-\frac{\sin (\beta-\alpha) \kappa_{H^{0}}^{f}}{2 s_{W} c_{W}\left|\kappa_{A^{0}}^{f}\right|} V_{4}^{p}\left(k^{2}, m_{f}, M_{Z}, m_{H^{0}}, m_{f}, v_{f},-a_{f}, 1,0\right)
\end{aligned}
$$




$$
\begin{aligned}
\Delta T_{14}= & \frac{m_{f} \cos 2 \beta \sin (\beta+\alpha) \kappa_{h^{0}}^{f}}{2 s_{W}^{2} c_{W}^{2}} V_{5}^{p}\left(k^{2}, m_{f}, M_{A}, m_{h^{0}}, m_{f}, 0,1,1,0\right) \\
\Delta T_{15}= & -\frac{m_{f} \cos 2 \beta \cos (\beta+\alpha) \kappa_{H^{0}}^{f}}{2 s_{W}^{2} c_{W}^{2}} V_{5}^{p}\left(k^{2}, m_{f}, M_{A}, m_{H^{0}}, m_{f}, 0,1,1,0\right) \\
\Delta T_{16}= & -\frac{m_{f} \sin 2 \beta \cos (\beta+\alpha) \kappa_{H^{0}}^{f} \kappa_{H^{0}}^{f^{\prime}}}{2 s_{W}^{2} c_{W}^{2} \kappa_{h^{0}}^{f}} V_{5}^{p}\left(k^{2}, m_{f}, M_{Z}, m_{H^{0}}, m_{f}, 0,1,1,0\right) \\
\Delta T_{17}= & \frac{m_{f} \sin 2 \beta \sin (\beta+\alpha) \kappa_{H^{0}}^{f^{\prime}}}{2 s_{W}^{2} c_{W}^{2}} V_{5}^{p}\left(k^{2}, m_{f}, M_{Z}, m_{h^{0}}, m_{f}, 0,1,1,0\right) \\
\Delta T_{18}= & -\frac{1}{2 s_{W}^{2} m_{f}\left|\kappa_{A^{0}}^{f}\right|} V_{5}^{p}\left(k^{2}, m_{f}, M_{W}, m_{H^{+}}, m_{f^{\prime}}, \nu_{f}, \pi_{f}, \lambda_{f}, \mu_{f}\right) \\
\Delta T_{19}= & \frac{m_{f}^{\prime}}{2 m_{f} s_{W}\left|\kappa_{A^{0}}^{f}\right|}\left\{\begin{array}{l}
\mu-A_{u} \cot \beta, f=d \\
\mu-A_{d} \tan \beta, f=u
\end{array}\right\} . \\
& \cdot \sum_{i=1}^{2} V_{5}^{p}\left(k^{2}, m_{f}, m_{\tilde{f}_{1}^{\prime}}, m_{\tilde{f}_{2}^{\prime}}, m_{\tilde{\chi}_{i}^{+}}, v_{i 1}^{f}+a_{i 1}^{f}, v_{i 1}^{f}-a_{i 1}^{f}, v_{i 2}^{f}-a_{i 2}^{f}, v_{i 2}^{f}+a_{i 2}^{f}\right) \\
\Delta T_{20}= & -\frac{m_{f}^{\prime}}{m_{f}\left|\kappa_{A^{0}}^{f}\right|}\left\{\begin{array}{l}
\mu-A_{u} \cot \beta, f=d \\
\mu-A_{d} \tan \beta, f=u
\end{array}\right\} . \\
& \cdot \sum_{i=1}^{4} V_{5}^{p}\left(k^{2}, m_{f}, m_{\tilde{f}_{1}}, m_{\tilde{f}_{2}}, m_{\tilde{\chi}_{i}^{0}}, v_{i 1}^{\prime f}+a_{i 1}^{\prime f}, v_{i 1}^{\prime f}-a_{i 1}^{\prime f}, v_{i 2}^{\prime f}-a_{i 2}^{\prime f}, v_{i 2}^{\prime f}+a_{i 2}^{\prime f}\right),
\end{aligned}
$$

where

$$
N_{j 1}^{\prime}=N_{j 1} c_{W}+N_{j 2} s_{W}, N_{j 2}^{\prime}=-N_{j 1} s_{W}+N_{j 2} c_{W} .
$$

The chargino and neutralino mass matrix $V_{i j}, U_{i j}, N_{i j}$ are given in appendix B. The vertex correction diagrams are described by the following functions with masses and couplings in its arguments.

$$
\begin{aligned}
& V_{1}^{s, p}\left(k^{2}, m_{f}, m_{1}, m_{2}, m_{3}, v, a\right)= \\
& \quad 4\left[\left( \pm m_{1}^{2}\left(v^{2}-a^{2}\right) \mp m_{f} m_{1}\left(v^{2}+a^{2}\right)+\left(m_{f}^{2}-k^{2} / 2\right)\left(v^{2}-a^{2}\right)\right) C_{0}\right. \\
& \quad+\left(m_{f} m_{1}(1 \pm 1)\left(v^{2}+a^{2}\right)-\left(4 m_{f}^{2}-k^{2}\right)\left(v^{2}-a^{2}\right)\right) C_{1}^{+}+\left(v^{2}-a^{2}\right) \\
& \left.\quad\left(4 C_{20}+\left(4 m_{f}^{2}-k^{2}\right) C_{2}^{+}+k^{2} C_{2}^{-}-1\right)\right]\left(k^{2}, m_{1}, m_{2}, m_{3}\right) \\
& V_{2}^{s, p}\left(k^{2}, m_{f}, m_{1}, m_{2}, m_{3}, v, a\right)= \\
& \quad\left[\left(\left(m_{f}^{2}+m_{1}^{2}\right)\left(v^{2}-a^{2}\right)+2 m_{f} m_{1}\left(v^{2}+a^{2}\right)\right) C_{0} \mp 4 m_{f}^{2}\left(v^{2}-a^{2}\right) C_{1}^{+}\right. \\
& \quad-2(1 \pm 1) m_{f} m_{1}\left(v^{2}+a^{2}\right) C_{1}^{+} \pm\left(v^{2}-a^{2}\right)\left(4 C_{20}+\left(4 m_{f}^{2}-k^{2}\right) C_{2}^{+}\right.
\end{aligned}
$$




$$
\begin{aligned}
& \left.\left.+k^{2} C_{2}^{-}-1 / 2\right)\right]\left(k^{2}, m_{1}, m_{2}, m_{3}\right) \\
& V_{3}^{s, p}\left(k^{2}, m_{f}, m_{1}, m_{2}, m_{3}, v, a, v^{\prime}, a^{\prime}, v^{\prime \prime}, a^{\prime \prime}\right)= \\
& -\left[\left(m_{f}^{2}\left( \pm v v^{\prime} a^{\prime \prime}+a a^{\prime} v^{\prime \prime}\right)+m_{f} m_{2}\left( \pm v a^{\prime} a^{\prime \prime}+a v^{\prime} v^{\prime \prime}\right)\right.\right. \\
& \left.+m_{f} m_{1}\left(v a^{\prime} v^{\prime \prime} \pm a v^{\prime} a^{\prime \prime}\right)+m_{1} m_{2}\left(v v^{\prime} v^{\prime \prime} \pm a a^{\prime} a^{\prime \prime}\right)\right) C_{0} \\
& -2 m_{f}\left(m_{2}\left( \pm v a^{\prime} a^{\prime \prime}+a v^{\prime} v^{\prime \prime}\right)+m_{1}\left(v a^{\prime} v^{\prime \prime}+a v^{\prime} a^{\prime \prime}\right)+\right. \\
& \left.2 m_{f}\left( \pm v v^{\prime} a^{\prime \prime}+a a^{\prime} v^{\prime \prime}\right)\right) C_{1}^{+}+\left(v v^{\prime} a^{\prime \prime} \pm a a^{\prime} v^{\prime \prime}\right)\left(4 C_{20}+\right. \\
& \left.\left.\left(4 m_{f}^{2}-k^{2}\right) C_{2}^{+}+k^{2} C_{2}^{-}-1 / 2\right)\right]\left(k^{2}, m_{1}, m_{2}, m_{3}\right) \\
& V_{4}^{s}\left(k^{2}, m_{f}, m_{1}, m_{2}, m_{3}, v, a, v^{\prime}, a^{\prime}\right)= \\
& {\left[2 m_{f} m_{3}\left(v v^{\prime}+a a^{\prime}\right)\left(C_{0}-2 C_{1}^{+}\right)+4\left(v v^{\prime}-a a^{\prime}\right)\right.} \\
& \left(\left(m_{f}^{2}-k^{2}\right) C_{1}^{+}-k^{2} C_{1}^{-}\right)-2\left(v v^{\prime}-a a^{\prime}\right)\left(4 C_{20}-\right. \\
& \left.\left.1 / 2+\left(4 m_{f}^{2}-k^{2}\right) C_{2}^{+}+k^{2} C_{2}^{-}\right)\right]\left(k^{2}, m_{1}, m_{2}, m_{3}\right) \\
& V_{4}^{p}\left(k^{2}, m_{f}, m_{1}, m_{2}, m_{3}, v, a, v^{\prime}, a^{\prime}\right)= \\
& {\left[6 m_{f} m_{3}\left(v a^{\prime}+a v^{\prime}\right) C_{0}+2\left(a v^{\prime}-v a^{\prime}\right)\left(4 C_{20}-\right.\right.} \\
& \left.\left.1 / 2+\left(4 m_{f}^{2}-k^{2}\right) C_{2}^{+}+k^{2} C_{2}^{-}\right)\right]\left(k^{2}, m_{1}, m_{2}, m_{3}\right) \\
& V_{5}^{s}\left(k^{2}, m_{f}, m_{1}, m_{2}, m_{3}, v, a, v^{\prime}, a^{\prime}\right)= \\
& -\left[2 m_{f}\left(v v^{\prime}-a a^{\prime}\right) C_{1}^{+}+m_{3}\left(v v^{\prime}+a a^{\prime}\right) C_{0}\right]\left(k^{2}, m_{1}, m_{2}, m_{3}\right) \\
& V_{5}^{p}\left(k^{2}, m_{f}, m_{1}, m_{2}, m_{3}, v, a, v^{\prime}, a^{\prime}\right)= \\
& -m_{3}\left(v a^{\prime}+a v^{\prime}\right) C_{0}\left(k^{2}, m_{1}, m_{2}, m_{3}\right) \\
& V_{6}^{s}\left(k^{2}, m_{f}, m_{1}, m_{2}, m_{3}, v, a, v^{\prime}, a^{\prime}\right)= \\
& -4\left[m_{3}\left(v v^{\prime}-a a^{\prime}\right) C_{0}-m_{f}\left(v v^{\prime}+a a^{\prime}\right) C_{1}^{+}\right]\left(k^{2}, m_{1}, m_{2}, m_{3}\right) \text {. }
\end{aligned}
$$

Fermion self energies are represented by the scalar functions $\Sigma_{S, V, A}^{f}$ through the decomposition in the scalar, vector and axialvector part:

$$
\Sigma^{f}(k)=\not k \Sigma_{V}^{f}\left(k^{2}\right)+\not k \gamma_{5} \Sigma_{A}^{f}\left(k^{2}\right)+m_{f} \Sigma_{S}^{f}\left(k^{2}\right)
$$

Standard model and genuine SUSY self energy contributions are listed separately in the following:

$$
\Sigma_{S, 2-H i g g s}^{f}\left(k^{2}\right)=-\frac{\alpha}{4 \pi}\left[\left(v_{f}^{2}-a_{f}^{2}\right)\left(4 B_{0}\left(k^{2}, m_{f}, M_{Z}\right)-2\right)-\frac{m_{f^{\prime}}^{2}}{2 s_{W}^{2} M_{W}^{2}}\right.
$$




$$
\begin{aligned}
& \left(B_{0}\left(k^{2}, m_{f^{\prime}}, M_{W}\right)+B_{0}\left(k^{2}, m_{f^{\prime}}, M_{H^{+}}\right)\right)-\frac{m_{f}^{2}}{4 s_{W}^{2} M_{W}^{2}} \\
& \left(\left(\kappa_{h^{0}}^{f}\right)^{2} B_{0}\left(k^{2}, m_{f}, M_{h^{0}}\right)+\left(\kappa_{H^{0}}^{f}\right)^{2} B_{0}\left(k^{2}, m_{f}, M_{H^{0}}\right)\right. \\
& \left.\left.-\left|\kappa_{A^{0}}^{f}\right|^{2} B_{0}\left(k^{2}, m_{f}, M_{A^{0}}\right)-B_{0}\left(k^{2}, m_{f}, M_{Z}\right)\right)\right] \\
& \Sigma_{S, S U S Y}^{f}\left(k^{2}\right)=\frac{\alpha}{4 \pi}\left[\sum_{i=1}^{2} \frac{m_{\tilde{\chi}_{i}^{+}}}{m_{f} s_{W}^{2}}\left(v_{i 1}^{f} a_{i 1}^{f} B_{0}\left(k^{2}, m_{\tilde{\chi}_{i}^{+}}, m_{\tilde{f}_{1}^{\prime}}\right)+v_{i 2}^{f} a_{i 2}^{f} B_{0}\left(k^{2}, m_{\tilde{\chi}_{i}^{+}}, m_{\tilde{f}_{2}^{\prime}}\right)\right)\right. \\
& \left.+\sum_{i=1}^{4} \frac{2 m_{\tilde{\chi}_{i}^{0}}}{m_{f}}\left(v_{i 1}^{\prime f} a_{i 1}^{f f} B_{0}\left(k^{2}, m_{\tilde{\chi}_{i}^{0}}, m_{\tilde{f}_{1}}\right)+v_{i 2}^{\prime f} a_{i 2}^{\prime f} B_{0}\left(k^{2}, m_{\tilde{\chi}_{i}^{0}}, m_{\tilde{f}_{2}}\right)\right)\right] \\
& \Sigma_{V, 2-\text { Higgs }}^{f}\left(k^{2}\right)=-\frac{\alpha}{4 \pi}\left[\left(v_{f}^{2}+a_{f}^{2}\right)\left(2 B_{1}\left(k^{2}, m_{f}, M_{Z}\right)+1\right)+\frac{1}{4 s_{W}^{2}}\right. \\
& \left(2 B_{1}\left(k^{2}, m_{f^{\prime}}, M_{W}\right)+1\right)+\frac{m_{f}^{2}}{4 s_{W}^{2} M_{W}^{2}}\left(\left(\kappa_{h^{0}}^{f}\right)^{2} B_{1}\left(k^{2}, m_{f}, M_{h^{0}}\right)\right. \\
& +\left(\kappa_{H^{0}}^{f}\right)^{2} B_{1}\left(k^{2}, m_{f}, M_{H^{0}}\right)+B_{1}\left(k^{2}, m_{f}, M_{Z}\right)+\left|\kappa_{A^{0}}^{f}\right|^{2} \\
& \left.B_{1}\left(k^{2}, m_{f}, M_{A^{0}}\right)\right)+\frac{1}{4 s_{W}^{2} M_{W}^{2}}\left(\left(m_{f}^{2}+m_{f^{\prime}}^{2}\right) B_{1}\left(k^{2}, m_{f^{\prime}}, M_{W}\right)\right. \\
& \left.\left.+\frac{m_{f}^{2} \tan ^{2} \beta+m_{f^{\prime}}^{2} \cot ^{2} \beta}{4 s_{W}^{2} M_{W}^{2}} B_{1}\left(k^{2}, m_{f^{\prime}}, m_{H^{+}}\right)\right)\right] \\
& \Sigma_{V, S U S Y}^{f}\left(k^{2}\right)=-\frac{\alpha}{4 \pi} \frac{1}{s_{W}^{2}}\left[\sum_{i=1}^{2} \frac{\left(v_{i 1}^{f}\right)^{2}+\left(a_{i 1}^{f}\right)^{2}}{2} B_{1}\left(k^{2}, m_{\tilde{\chi}_{i}^{+}}, m_{\tilde{f}_{1}^{\prime}}\right)+\frac{\left(v_{i 2}^{f}\right)^{2}+\left(a_{i 2}^{f}\right)^{2}}{2}\right. \\
& B_{1}\left(k^{2}, m_{\tilde{\chi}_{i}^{+}}, m_{\tilde{f}_{2}^{\prime}}\right)+2 s_{W}^{2} \sum_{i=1}^{4}\left(\frac{\left(v_{i 1}^{\prime f}\right)^{2}+\left(a_{i 1}^{\prime f}\right)^{2}}{2}\right. \\
& \left.\left.B_{1}\left(k^{2}, m_{\tilde{\chi}_{i}^{+}}, m_{\tilde{f}_{1}}\right)+\frac{\left(v_{i 2}^{\prime f}\right)^{2}+\left(a_{i 2}^{\prime f}\right)^{2}}{2} B_{1}\left(k^{2}, m_{\tilde{\chi}_{i}^{+}}, m_{\tilde{f}_{2}}\right)\right)\right] \\
& \Sigma_{A, 2-H i g g s}^{f}\left(k^{2}\right)=-\frac{\alpha}{4 \pi}\left[-2 v_{f} a_{f}\left(2 B_{1}\left(k^{2}, m_{f}, M_{Z}\right)+1\right)-\frac{1}{4 s_{W}^{2}}\right. \\
& \left(2 B_{1}\left(k^{2}, m_{f^{\prime}}, M_{W}\right)-1\right)+\frac{1}{4 s_{W}^{2} M_{W}^{2}}\left(\left(m_{f^{\prime}}^{2}-m_{f}^{2}\right) B_{1}\left(k^{2}, m_{f^{\prime}}, M_{W}\right)\right. \\
& \left.\left.+\left(m_{f}^{2} \tan ^{2} \beta-m_{f^{\prime}}^{2} \cot ^{2} \beta\right) B_{1}\left(k^{2}, m_{f^{\prime}}, m_{H^{+}}\right)\right)\right] \\
& \Sigma_{A, S U S Y}^{f}\left(k^{2}\right)=-\frac{\alpha}{4 \pi} \frac{1}{s_{W}^{2}}\left[\sum _ { i = 1 } ^ { 2 } \left(\frac{-\left(v_{i 1}^{f}\right)^{2}+\left(a_{i 1}^{f}\right)^{2}}{2} B_{1}\left(k^{2}, m_{\tilde{\chi}_{i}^{+}}, m_{\tilde{f}_{1}^{\prime}}\right)+\frac{-\left(v_{i 2}^{f}\right)^{2}+\left(a_{i 2}^{f}\right)^{2}}{2}\right.\right. \\
& \left.B_{1}\left(k^{2}, m_{\tilde{\chi}_{i}^{+}}, m_{\tilde{f}_{2}^{\prime}}\right)\right)+2 s_{W}^{2} \sum_{i=1}^{4}\left(\frac{-\left(v_{i 1}^{\prime f}\right)^{2}+\left(a_{i 1}^{\prime f}\right)^{2}}{2} B_{1}\left(k^{2}, m_{\tilde{\chi}_{i}^{0}}, m_{\tilde{f}_{1}}\right)\right. \\
& \left.\left.+\frac{-\left(v_{i 2}^{\prime f}\right)^{2}+\left(a_{i 2}^{\prime f}\right)^{2}}{2} B_{1}\left(k^{2}, m_{\tilde{\chi}_{i}^{0}}, m_{\tilde{f}_{2}}\right)\right)\right] \text {. }
\end{aligned}
$$


The 2-point functions $B_{0}, B_{1}$ are defined

$$
\begin{aligned}
& B_{0}\left(k^{2}, m_{1}, m_{2}\right)=\Delta-\int_{0}^{1} d x \log \frac{x^{2} k^{2}-x\left(k^{2}+m_{1}^{2}-m_{2}^{2}\right)+m_{1}^{2}-i \epsilon}{\mu^{2}}, \\
& B_{1}\left(k^{2}, m_{1}, m_{2}\right)=-\frac{k^{2}+m_{1}^{2}-m_{2}^{2}}{2 k^{2}} B_{0}\left(k^{2}, m_{1}, m_{2}\right)+\frac{m_{1}^{2}-m_{2}^{2}}{2 k^{2}} B_{0}\left(0, m_{1}, m_{2}\right),
\end{aligned}
$$

where

$$
\Delta=\frac{2}{\varepsilon}-\gamma+\log 4 \pi, \quad \varepsilon=4-D
$$

and the mass scale $\mu$ are the UV-parameters from dimensional regularization in Ddimensions.

The 3-point functions $C_{0}, C_{1}^{+,-}, C_{2}^{0,+,-}$ are for equal external fermion masses $p^{2}=$ $p^{\prime 2}=m_{f}^{2}$ :

$$
\frac{i}{(4 \pi)^{2}} C_{0}\left(k^{2}, m_{1}, m_{2}, m_{3}\right)=\int \frac{d^{4} k}{(2 \pi)^{4}} \frac{1}{D_{1} D_{2} D_{3}},
$$

with $k^{2}=\left(p-p^{\prime}\right)^{2}$ and the denominators

$$
\begin{aligned}
& D_{1}=\left(k-p^{\prime}\right)^{2}-m_{1}^{2}+i \epsilon \\
& D_{2}=(k-p)^{2}-m_{2}^{2}+i \epsilon \\
& D_{3}=k^{2}-m_{3}^{2}+i \epsilon
\end{aligned}
$$

and for different masses $m_{1}, m_{2}, m_{3}$ :

$$
\begin{aligned}
\left(p+p^{\prime}\right)^{2} C_{1}^{+}\left(k^{2}, m_{1}, m_{2}, m_{3}\right)= & B_{0}\left(k^{2}, m_{1}, m_{2}\right)-\frac{B_{0}\left(m_{f}^{2}, m_{1}, m_{3}\right)+B_{0}\left(m_{f}^{2}, m_{2}, m_{3}\right)}{2}+ \\
& +\frac{2 m_{f}^{2}+2 m_{3}^{2}-m_{1}^{2}-m_{2}^{2}}{2} C_{0} \\
2 k^{2} C_{1}^{-}\left(k^{2}, m_{1}, m_{2}, m_{3}\right)= & B_{0}\left(m_{f}^{2}, m_{1}, m_{3}\right)-B_{0}\left(m_{f}^{2}, m_{2}, m_{3}\right)+\left(m_{2}^{2}-m_{1}^{2}\right) C_{0} \\
4 C_{2}^{0}\left(k^{2}, m_{1}, m_{2}, m_{3}\right)= & B_{0}\left(k^{2}, m_{1}, m_{2}\right)+\left(m_{1}^{2}+m_{2}^{2}-2 m_{3}^{2}-2 m_{f}^{2}\right) C_{1}^{+} \\
& +\left(m_{1}^{2}-m_{2}^{2}\right) C_{1}^{-}+2 m_{3}^{2} C_{0}+1 \\
\left(p+p^{\prime}\right)^{2} C_{2}^{+}\left(k^{2}, m_{1}, m_{2}, m_{3}\right)= & \frac{1}{4}\left[B_{1}\left(m_{f}^{2}, m_{3}, m_{2}\right)+B_{1}\left(m_{f}^{2}, m_{3}, m_{1}\right)+2 B_{0}\left(k^{2}, m_{1}, m_{2}\right)\right. \\
& \left.+2\left(2 m_{3}^{2}-m_{1}^{2}-m_{2}^{2}+2 m_{f}^{2}\right) C_{1}^{+}\right]-C_{2}^{0} \\
k^{2} C_{2}^{-}\left(k^{2}, m_{1}, m_{2}, m_{3}\right)= & -\frac{1}{4}\left(B_{1}\left(m_{f}^{2}, m_{3}, m_{2}\right)+B_{1}\left(m_{f}^{2}, m_{3}, m_{1}\right)\right) \\
& +2\left(m_{1}^{2}-m_{2}^{2}\right) C_{1}^{-}-C_{2}^{0} .
\end{aligned}
$$

The analytic expression for the scalar vertex integral $C_{0}$ can be found in 28 . 


\section{B Gaugino mass matrix}

The chargino $2 \times 2$ mass matrix is given by

$$
\mathcal{M}_{\tilde{\chi}^{ \pm}}=\left(\begin{array}{ll}
M & M_{W} \sqrt{2} \sin \beta \\
M_{W} \sqrt{2} \cos \beta & -\mu
\end{array}\right)
$$

with the SUSY soft breaking parameters $\mu$ and $M$ in the diagonal matrix elements. The physical chargino mass states $\tilde{\chi}_{i}^{ \pm}$are the rotated wino and charged Higgsino states:

$$
\begin{aligned}
& \tilde{\chi}_{i}^{+}=V_{i j} \psi_{j}^{+} \\
& \tilde{\chi}_{i}^{-}=U_{i j} \psi_{j}^{-} ; i, j=1,2 .
\end{aligned}
$$

$V_{i j}$ and $U_{i j}$ are unitary chargino mixing matrices obtained from the diagonalization of the mass matrix (B.1):

$$
\mathrm{U}^{*} \mathcal{M}_{\tilde{\chi}^{ \pm}} \mathrm{V}^{-1}=\operatorname{diag}\left(\mathrm{m}_{\tilde{\chi}_{1}^{ \pm}}^{2}, \mathrm{~m}_{\tilde{\chi}_{2}^{ \pm}}^{2}\right)
$$

The neutralino $4 \times 4$ mass matrix yields:

$$
\mathcal{M}_{\tilde{\chi}^{0}}=\left(\begin{array}{cccc}
M^{\prime} & 0 & -M_{Z} \sin \theta_{W} \cos \beta & M_{Z} \sin \theta_{W} \sin \beta \\
0 & M & M_{Z} \cos \theta_{W} \cos \beta & -M_{Z} \cos \theta_{W} \sin \beta \\
-M_{Z} \sin \theta_{W} \cos \beta & M_{Z} \cos \theta_{W} \cos \beta & 0 & \mu \\
M_{Z} \sin \theta_{W} \sin \beta & -M_{Z} \cos \theta_{W} \sin \beta & \mu & 0
\end{array}\right)
$$

where the diagonalization introduces the unitary matrix $N_{i j}$ by:

$$
\mathrm{N}^{*} \mathcal{M}_{\tilde{\chi}^{0}} \mathrm{~N}^{-1}=\operatorname{diag}\left(\mathrm{m}_{\tilde{\chi}_{\mathrm{i}}^{0}}\right)
$$




\section{References}

[1] H.P. Nilles, Phys. Rep. 110 (1984) 1

H.E. Haber, G. Kane, Phys. Rep. 117 (1985) 75

J.F. Gunion, H.E. Haber, Nucl. Phys. B 272 (1986) 1; Nucl. Phys. B 402 (1993) 567

J.F. Gunion, H.E. Haber, G. Kane, S. Dawson: The Higgs Hunter's Guide, AddisonWesley 1990

[2] J. Ellis, S. Kelly, D. Nanopoulos, Phys. Lett. B 249 (1990) 441

U. Amaldi, W.de. Boer, H. Fürstenau, Phys. Lett. B 260 (1991) 447

P. Langacker, M. Luo, Phys. Rev. D 44 (1991) 477

[3] L. Hall, L. Randall, Phys. Rev. Lett. 65 (1990) 2939

[4] H.E. Haber, R. Hempfling, Phys. Rev. Lett. 66 (1991) 1815

[5] Y. Okada, M. Yamaguchi, T. Yanagida, Prog. Theor. Phys. 85 (1991) 1

D. Pierce, A. Papadopoulos, S. Johnson, Phys. Rev. Lett. 68 (1992) 3678

[6] R. Barbieri, M. Frigeni, Phys. Lett. 258 B (1991) 395

R. Barbieri, M. Frigeni, F. Caravaglios, Phys. Lett. 258 B (1991) 167

J.R. Espinosa, M.Quiros, Phys. Lett. 266 (1991) 389

K. Sasaki, M. Carena, C.E.M. Wagner, Phys. Rev. Lett. 381 B (1992) 66

H.E. Haber, R. Hempfling, Phys. Rev. D 48 (1993) 4280

[7] J. Ellis, G. Ridolfi, F. Zwirner, Phys. Lett. 257 B (1991) 83

J. Ellis, G. Ridolfi, F. Zwirner, Phys. Lett. 262 B (1991) 477

[8] Y. Okada, M. Yamaguchi, T. Yanagida, Phys. Lett. 262 B (1991) 54

J.L. Lopez, D.V. Nanopoulos, Phys. Lett. 266 B (1991) 397

A. Brignole, J. Ellis, G. Ridolfi, F. Zwirner, Phys. Lett. 271 B (1991) 123

[9] A. Yamada, Phys. Lett. 263 B (1991) 233

M.A. Diaz, H.E. Haber, Phys. Rev. D 45 (1992) 4246

M.A. Diaz, H.E. Haber, Phys. Rev. D 46 (1992) 3086

A. Brignole, Phys. Lett. 281 B (1992) 284

A. Brignole, Phys. Lett. 277 B (1992) 313

D. Pierce, A. Papadopoulos, Phys. Rev. D 47 (1992) 222

A. Yamada, Z. Phys. C 61 (1994) 247 
[10] J. Kodaira, Y. Yasui, K. Sasaki, Hiroshima preprint HUPD-9316, YNU-HEPTh93-102 (Nov. 1993)

R. Hempfling, A. H. Hoang, Phys. Lett. B 331 (1994) 99

J.A. Casas, J.R. Espinosa, M. Quiros, A. Riotto, CERN-TH. 7334/94 (July 1994)

[11] P.H. Chankowski, S. Pokorski, J. Rosiek, Phys. Lett. 274 B (1992) 191

P.H. Chankowski, S. Pokorski, J. Rosiek, Nucl. Phys. 423 B (1994) 437

[12] A. Dabelstein, Karlsruhe preprint KA-THEP-5-1994, to appear in Z. Phys. C

[13] P.H. Chankowski, S. Pokorski, J. Rosiek, Nucl. Phys. 423 B (1994) 497

[14] E. Braaten, J.P. Leveille, Phys. Rev. D 22 (1980) 715

N. Sakai, Phys. Rev. D 22 (1980) 2220

T. Inami, T. Kubota, Nucl. Phys. B 179 (1981) 171

[15] D.Yu. Bardin, B.M. Vilensky, P.Ch. Christova, Yad. Fiz. 53 (1991) 240 (in russian)

[16] M. Drees, K. Hikasa, Phys. Lett. 240 B (1990) 455

[17] A. Djouadi, M. Spira, P.M. Zerwas, Phys. Lett. B 276 (1992) 350

[18] A. Djouadi, M. Spira, J.J. van der Bij, P.M. Zerwas, Phys. Lett. B 257 (1991) 187

[19] J.Gunion, H.Haber, Nucl. Phys. B 307 (1988) 445

[20] K. Ng, H. Pois, T.C. Yuan, Phys. Rev. D 40 (1989) 1689

A. Djouadi, M. Drees, Madison-preprint MADPH-94-853, October 1994

[21] A. Sirlin, Phys. Rev. D 22 (1980) 971

W.J. Marciano, A. Sirlin, Phys. Rev. D 22 (1980) 2695

[22] D. Garcia, J. Solà, Mod. Phys. Lett. A 9 (1994) 211

P.H. Chankowski, A. Dabelstein, W. Hollik, W. Mösle, S. Pokorski, J. Rosiek, Nucl. Phys. B 417 (1994) 101

[23] Z. Kunszt, F. Zwirner, Nucl. Phys. B 385 (1992) 3

[24] M.A. Diaz, Vanderbilt-preprint VAND-TH-94-19, August 1994, presented at the Eight Meeting of the Division of Particles and Fields of the American Physical Society DPF '94, The University of New Mexico, Albuquerque NM, August 2-6, 1994 
[25] D.R.T. Jones, Phys. Rev. D 25 (1984) 581

O.V. Tarasov, A.A. Vladimirov, A.Y. Zharkov, Phys. Lett 93 B (1980) 429

S.G. Gorishny, A.L. Kataev, S. Larin, Sov. J. Nucl. Phys. 40 (1984) 329

D.V. Nanopoulos, D.A. Ross, Nucl. Phys. B 157 (1979) 273

R. Tarrach, Nucl. Phys. B 183 (1981) 384

[26] F. Abe et al., CDF Collaboration, FERMILAB-PUB-95-022-E, March 1995

S. Abachi et al., D0 Collaboration, FERMILAB-PUB-95-028-E, March 1995

[27] A. Dabelstein, W. Hollik, Z. Phys. C 53 (1992) 507

B. Kniehl, Nucl. Phys. B 376 (1992) 3

[28] W. Hollik, Fortschr. Phys. 38 (1990) 165 


\section{FIGURE CAPTIONS}

Figure 1. The mixing angle $\sin ^{2} \alpha$ as a function of the physical light Higgs mass $M_{h^{0}}$ for $\tan \beta=2,30$ in Fig. 1a) and $\tan \beta=0.5,8$ in Fig. 1b). Figs. 1 c,d) show the same data for $\sin ^{2} \alpha$ as a function of the pseudoscalar mass $M_{A^{0}}$. The solid line is $\sin ^{2} \alpha_{\text {eff }}$ in the effective potential approximation of Eq. (3.14). $\sin ^{2} \alpha_{1-l o o p}$ in the full one-loop calculation of Eq. (3.11) is shown by the dotted line and $\sin ^{2} \alpha_{b}$ in Eq. (3.16) with the non-universal $h^{0} \rightarrow b \bar{b}$ vertex correction is the dashed result. The parameters in Fig. 1 are $m_{t}=175 \mathrm{GeV}, m_{s f}=700 \mathrm{GeV}, \mu=100 \mathrm{GeV}, M=550 \mathrm{GeV}$, no sfermion left-right mixing.

Figure 2. Figs. 2a,b) show the decay width $\Gamma_{h^{0} \rightarrow b \bar{b}}$, including the full weak MSSM oneloop corrections as a function of the physical light MSSM Higgs mass $M_{h^{0}}$ for $\tan \beta=2$, 30 in Fig. 2a) and $\tan \beta=0.5,8$ in Fig. $2 b$ ). $m_{t}=175 \mathrm{GeV}$ (solid line), $m_{t}=160 \mathrm{GeV}$ (dotted line) and $m_{t}=190 \mathrm{GeV}$ (dashed line). No sfermion mixing. The standard Higgs decay width $\Gamma_{h^{0} \rightarrow b \bar{b}}$ with one-loop electroweak corrections is labeled in Fig. 2. Figs. 2c,d) contain the data of Figs. 2a,b) as a function of the pseudoscalar Higgs mass $M_{A^{0}}$. The standard Higgs mass is chosen to be equal the physical light Higgs mass $M_{h^{0}}$.

Figure 3. The $h^{0} \rightarrow b \bar{b}$ decay width dependence on the sfermion masses is plotted in Figs. 3a,b) as a function of the physical light MSSM Higgs mass $M_{h^{0}} \cdot \tan \beta=2,30$ in Fig. 3a) and $\tan \beta=0.5,8$ in Fig. 3b). All sfermion soft breaking parameters are equal, $m_{s f}=1 \mathrm{TeV}$ (solid line), $m_{s f}=500 \mathrm{GeV}$ (dotted line), $m_{s f}=300 \mathrm{GeV}$ (short dashed line) and $m_{s f}=200 \mathrm{GeV}$ (long dashed line) and no sfermion mixing is assumed. In Fig. 3c) mixing effects of sfermions are shown for $\tan \beta=2,30 . A_{t}^{\prime}=0$ (solid line), $A_{t}^{\prime}=100 \mathrm{GeV}$ (dotted line), $A_{t}^{\prime}=200 \mathrm{GeV}$ (short dashed), $A_{t}^{\prime}=300 \mathrm{GeV}$ (long dashed) and $A_{t}^{\prime}=400 \mathrm{GeV}$ (dot dashed), see Eq. (4.14). Gaugino contributions to the decay width $h^{0} \rightarrow b \bar{b}$ are plotted in Fig. 3d) for $M=100 \mathrm{GeV}$ (dotted) and $M=200 \mathrm{GeV}$ (dashed). The $\mu$ parameters are described in the figure.

Figure 4. Figs. 4 a,b) show the $H^{0} \rightarrow b \bar{b}$ decay width with full MSSM one-loop contributions for $\tan \beta=0.5,2,8,30$ as a function of the physical heavy Higgs mass $M_{H^{0}}$. In Fig. 4a) the top-quark mass is $m_{t}=175 \mathrm{GeV}$ (solid line), $m_{t}=160 \mathrm{GeV}$ (dotted line) and $m_{t}=190 \mathrm{GeV}$ (dashed line). The soft breaking parameters are $m_{s f}=700$ $\mathrm{GeV}, \mu=100 \mathrm{GeV}, M=550 \mathrm{GeV}$. Fig. 4b) shows the dependence on sfermion masses for a constant top-quark mass $m_{t}=175 \mathrm{GeV}$. The lines correspond to $m_{s f}=1 \mathrm{TeV}$ 
(solid), $m_{s f}=500 \mathrm{GeV}$ (dotted), $m_{s f}=300 \mathrm{GeV}$ (short dashed), $m_{s f}=200 \mathrm{GeV}$ (long dashed). The pseudoscalar $A^{0} \rightarrow b \bar{b}$ decay width is plotted in Fig. 4c) as a function of the pseudoscalar mass $M_{A^{0}}$ with full MSSM one-loop contributions. Fig. 4d) shows the $A^{0} \rightarrow b \bar{b}$ vertex corrections $\delta \Gamma_{b}=2 \Re e \Delta T_{A^{0}}$, Eq. (3.4), for $\tan \beta=0.5,2,30$ and top-quark masses $m_{t}=175 \mathrm{GeV}$ (solid line), $m_{t}=160 \mathrm{GeV}$ (dotted line), $m_{t}=190 \mathrm{GeV}$ (dashed line). In Figs. 4 c,d) $m_{s f}=700 \mathrm{GeV}, \mu=100 \mathrm{GeV}, M=550 \mathrm{GeV}$.

Figure 5. Gluino contributions to the one-loop vertex corrections are plotted in Figs. 5 a,b) for the $h^{0} \rightarrow b \bar{b}$ decay width, Figs. 5 c,d ) for $H^{0} \rightarrow b \bar{b}$ and in Figs. 5 e,f) for $A^{0} \rightarrow b \bar{b}$. In Figs. 5 a,c,e) the $H \rightarrow b \bar{b}$ decay width is plotted as a function of the respective Higgs mass. $\tan \beta=0.5,2,8,30$ with a gluino mass $m_{g l}=500 \mathrm{GeV}$ (solid line), $m_{g l}=200 \mathrm{GeV}$ (dotted line). Sfermion soft breaking masses are $m_{s f}=700 \mathrm{GeV}$ (solid line) and $m_{s f}=500 \mathrm{GeV}$ (dotted line), $m_{t}=175 \mathrm{GeV}, \mu=100 \mathrm{GeV}, M=550$ $\mathrm{GeV}$. Figs. $5 \mathrm{~b}, \mathrm{~d}, \mathrm{f})$ show the $\mu$ parameter dependence on the $H \rightarrow b \bar{b}$ vertex corrections for pseudoscalar masses $M_{A^{0}}=50 \mathrm{GeV}, 150 \mathrm{GeV}, 250 \mathrm{GeV} \cdot \tan \beta=8, m_{t}=175 \mathrm{GeV}$, $m_{g l}=200 \mathrm{GeV}, m_{s f}=500 \mathrm{GeV}, M=550 \mathrm{GeV}$ and $A_{f}=0$.

Figure 6. The light Higgs boson decay branching ratios $h^{0} \rightarrow f \bar{f}$ for the decay channels $f=b$ (Fig. 6 a), $\tau, c$ (Fig. 6 b) with full one-loop MSSM contributions (solid line) and in the approximation of Eq. (3.14) (dashed). The branching ratios are plotted as functions of the physical light Higgs mass $M_{h^{0}}$ for $\tan \beta=0.5,2,8,30$. The dotted curves are the standard Higgs branching ratios. $m_{t}=175 \mathrm{GeV}, m_{s f}=700 \mathrm{GeV}, \mu=100 \mathrm{GeV}$, $M=550 \mathrm{GeV}$, no sfermion left-right mixing. 

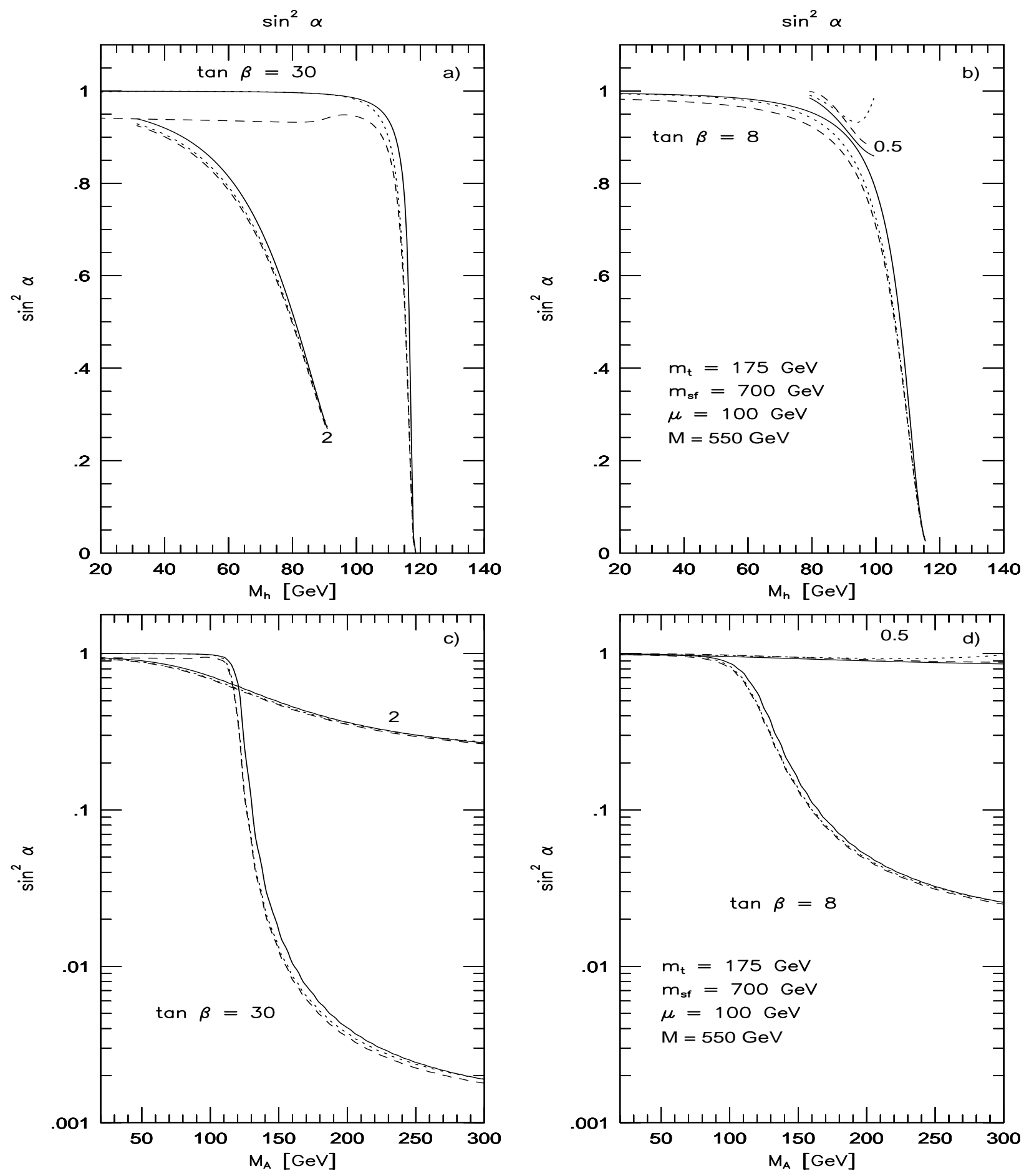

Figure 1: 

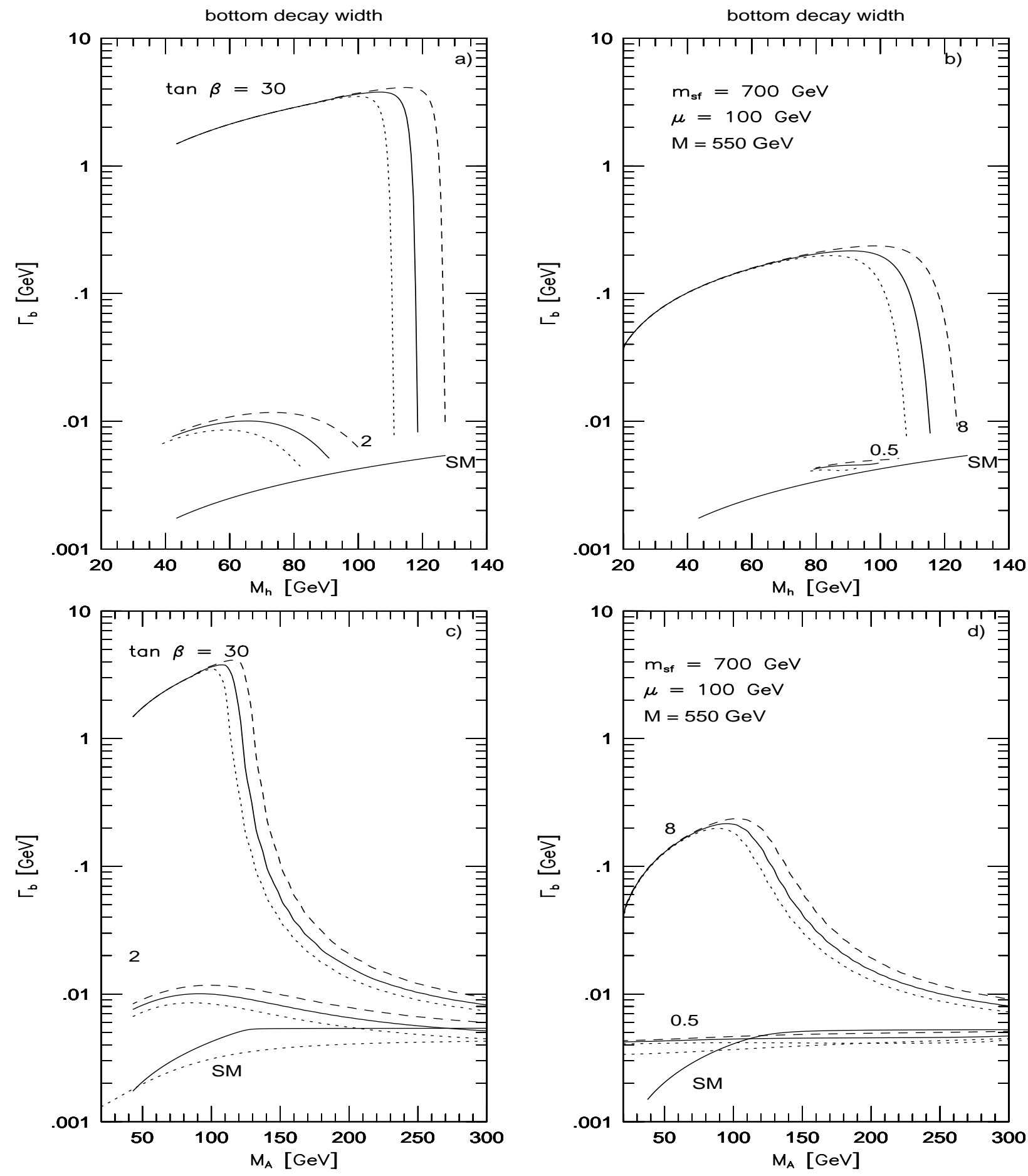

Figure 2: 

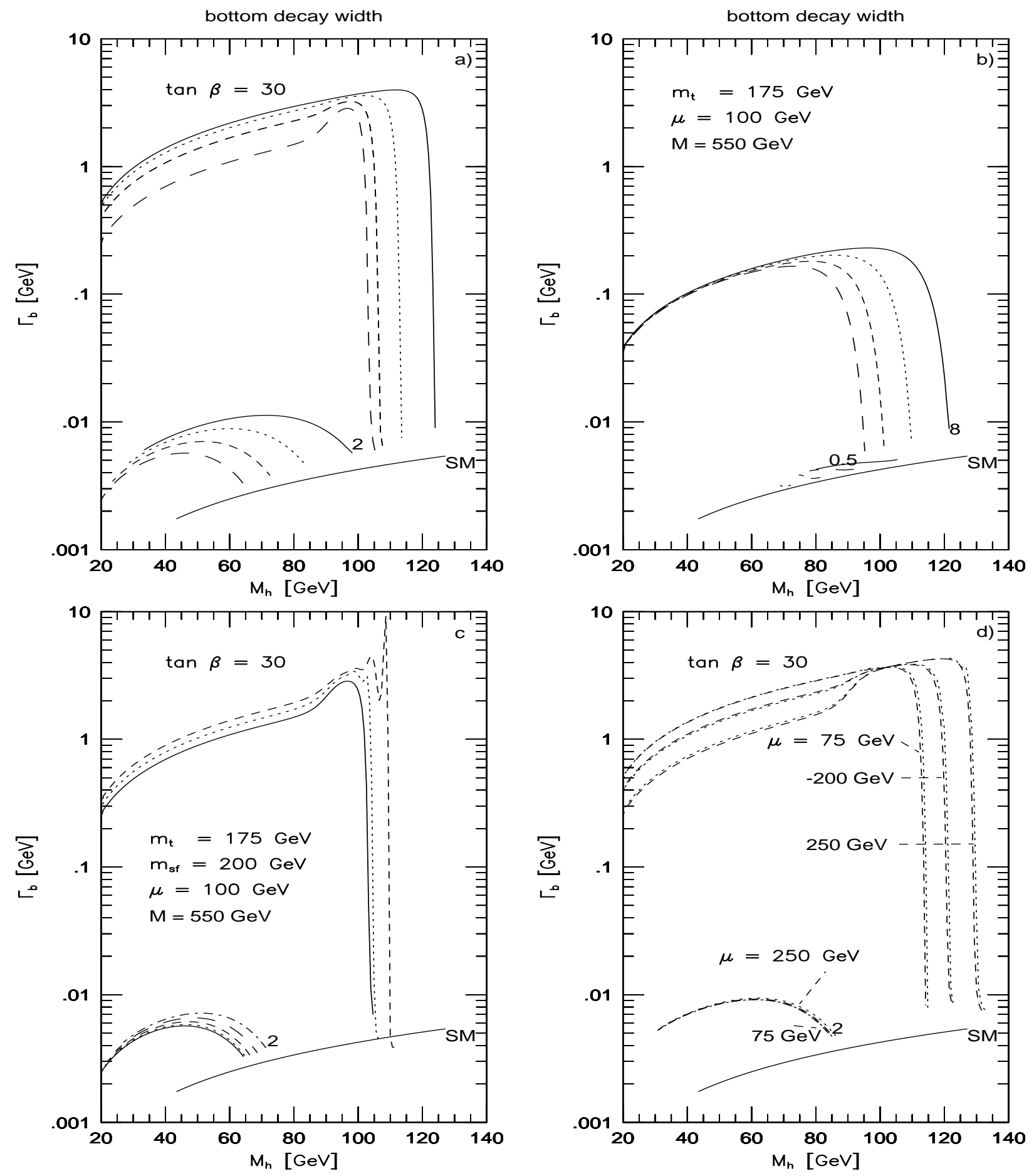

Figure 3: 

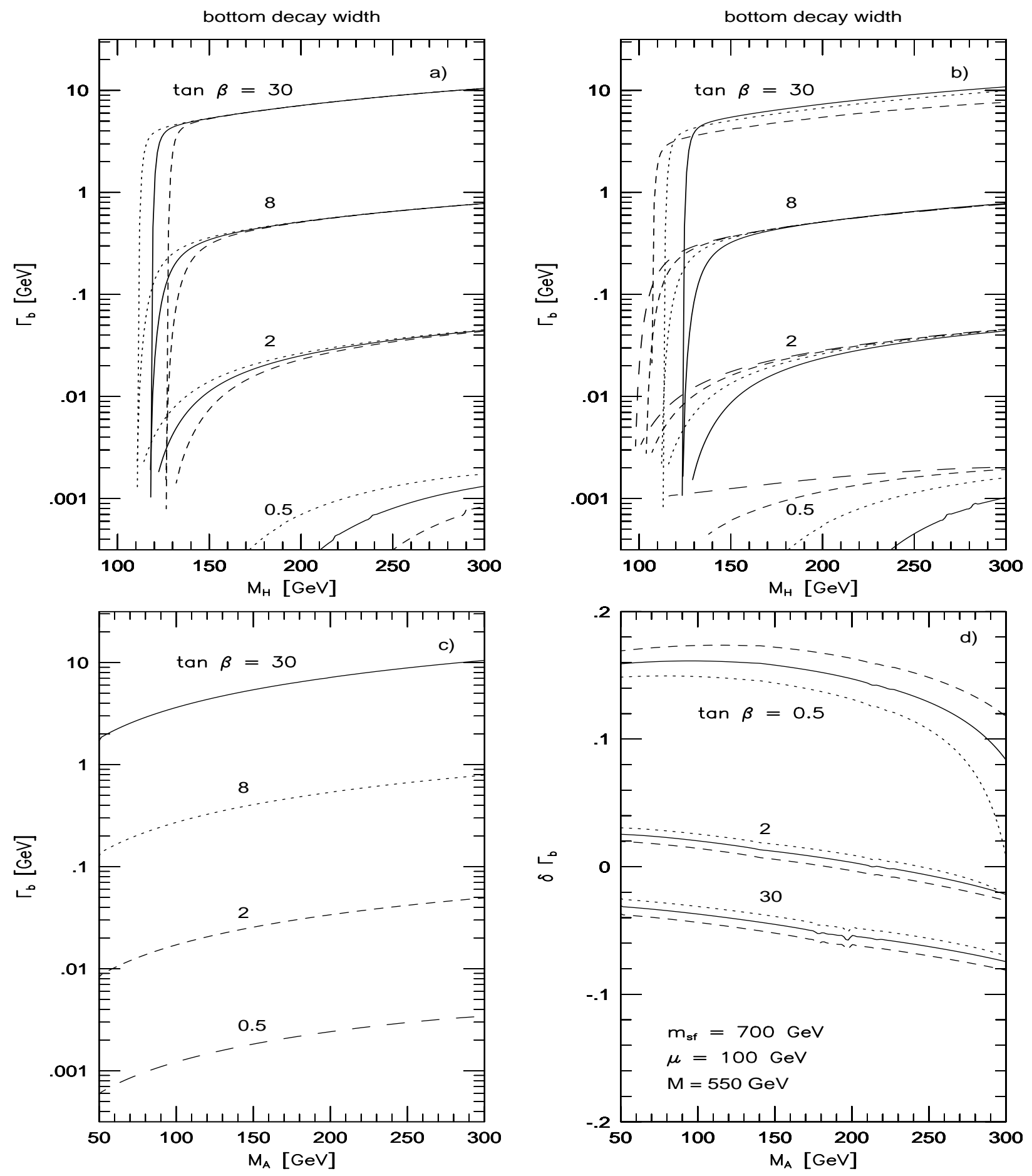

Figure 4: 

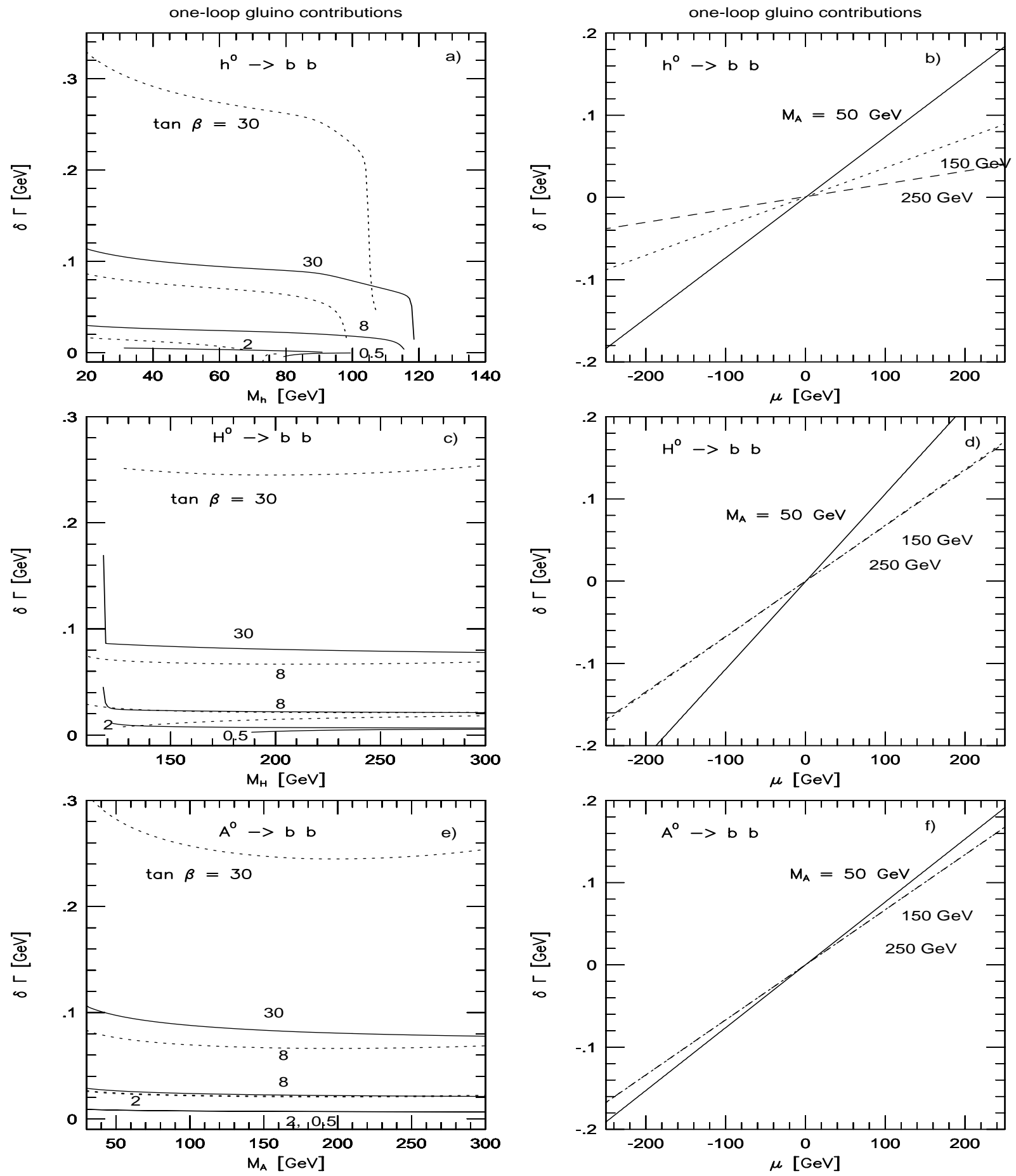

Figure 5: 

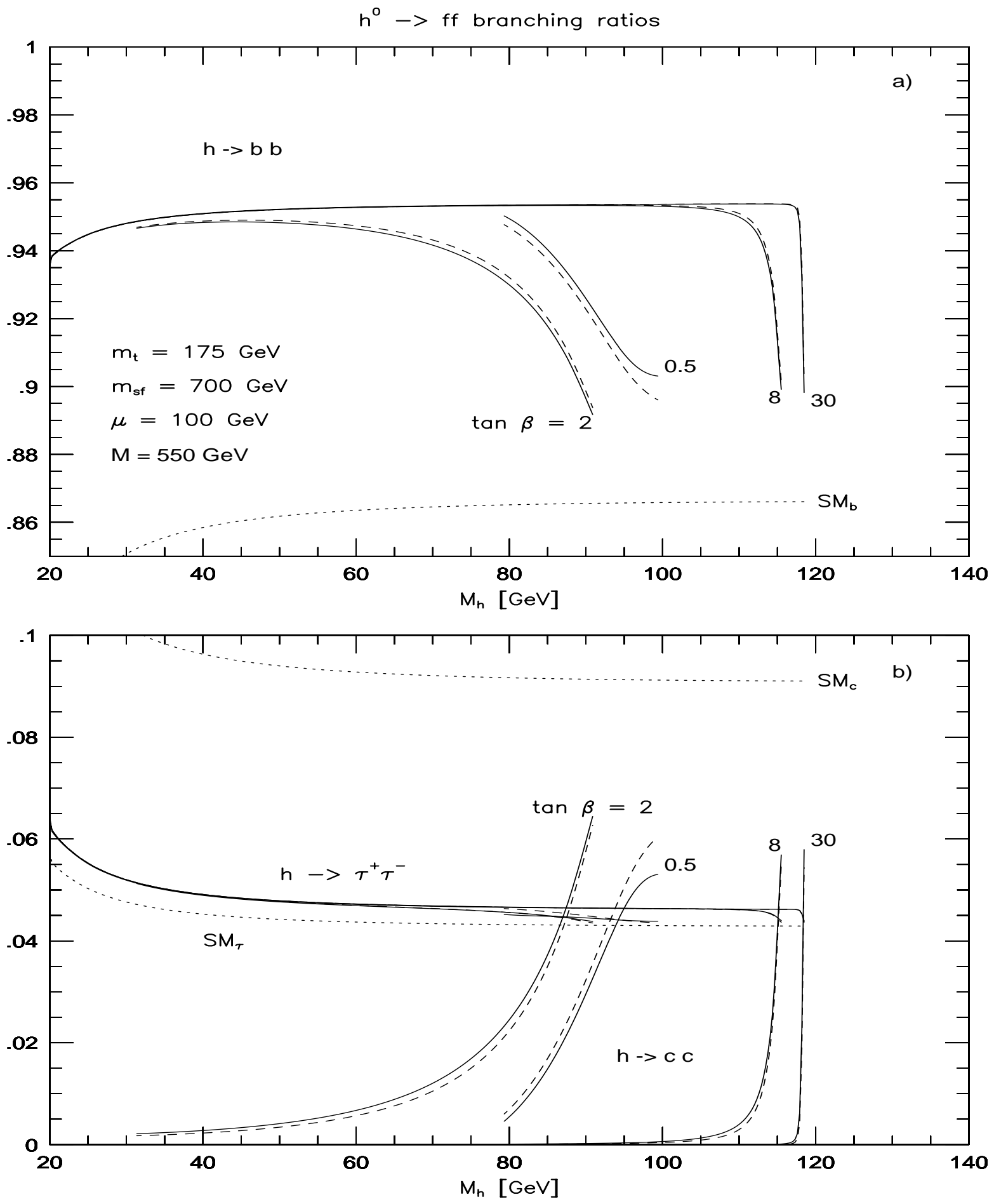

Figure 6: 